\title{
VASCULAR FLORA OF THE TOWN OF BLAGAJ (SOUTH BOSNIA AND HERZEGOVINA)
}

\author{
Semir Maslo ${ }^{1}$ \& Sabaheta Abadžić ${ }^{2}$ \\ 1Lundåkerskolan Gislaved Sweden (semmas@edu.gislaved.se) \\ ${ }^{2}$ National Museum of Bosnia and Herzegovina (sabahetaabadzic@yahoo.com)
}

Maslo, S. \& Abadžić, S.: Vascular flora of the town of Blagaj (south Bosnia and Herzegovina). Nat. Croat., Vol. 24, No. 1, 59-92, 2015, Zagreb.

The flora of the town of Blagaj was observed and researched during the vegetation seasons from 2004 to 2014. In total, 723 plant taxa from 102 families are presented in the list, 577 of which are newly found, while the presence of 14 previously reported taxa was not confirmed. Alien taxa present exclusively in culture were not analysed in this work. The most common family is Asteraceae s. l. (11.76\%), followed by Poaceae $(9.82 \%)$, Fabaceae $(7.75 \%)$ and Lamiaceae $(6.50 \%)$. The predominant life-forms are therophytes and hemicryptophytes with 265 and 264 taxa, respectively (36.65\% and 36.51\%). The floral elements spectrum shows the strong prevalence of Mediterranean plants $(29.46 \%)$, while Central European plants are almost absent $(0.41 \%)$. A total of 38 taxa are found on the National Red List, while 27 taxa are classified as invasive.

Keywords: vascular plants, biodiversity, SE Europe

Maslo, S. \& Abadžić, S.: Vaskularna flora grada Blagaja (južna Bosna i Hercegovina). Nat. Croat., Vol. 24, No. 1, 59-92, 2015, Zagreb.

U razdoblju između 2004. i 2014. godine izvršena su floristička istraživanja na području naselja Blagaj. U rezultatima su navedene ukupno 723 biljne svojte iz 102 porodice, od kojih se 557 prvi put navodi za istraženo područje, dok 14 prethodno zabilježenih svojti nije potvrđeno u ovom istraživanju. Porodice s najvećim brojem svojta su Asteraceae s. l. (11,76\%), Poaceae (9,82\%), Fabaceae (7,75\%) i Lamiaceae $(6,50 \%)$. Terofiti su dominantni životni oblik $(36,65 \%)$, dok su mediteranske biljke najzastupljenije (29,46\%). Na Spisku biljnih vrsta za Crvenu knjigu BiH kao i na popisu Crvene liste flore Federacije $\mathrm{BiH}$, nalazi se 38 svojti, dok je invazivnih vrsta zabilježeno ukupno 27.

Ključne riječi: vaskularne biljke, biodiverzitet, JI Europa

\section{INTRODUCTION}

Blagaj is a small town situated at the source of the Buna River, at an altitude of $70 \mathrm{~m}$, $12 \mathrm{~km}$ southeast of Mostar at the edge of Bišće Plain, in the south part of Bosnia and Herzegovina (Fig. 1). A unique natural landscape is formed by the stone cliff and the Buna River and includes the medieval fort known as Stjepan grad as well as the Derviš Tekke from the Ottoman period. The oldest written testament to the existence of medieval Blagaj dates back to the 10th century. The archaeological material found above the slopes of hills indicates that settlements existed here during the prehistoric and Roman periods. According to the 2013 census, Blagaj had a population of 2684 inhabitants.

The geology consists mostly of Cretaceous and Eocene limestone (Hrvatović, 2006). The pedological substrate consists mostly of terra rossa and brown soils on limestone and rendzina soils. The climate is sub-Mediterranean, with short mild winters, usually with- 
out snow, and long hot summers. As stated in the Biogeographic Map of Europe (RIvAsMARTínez et al., 2004), Blagaj is in the Euro-Siberian Region, Alpine-Caucasaon Subregion, Apennine-Balkan Province, and Illyrian sector. With respect to natural vegetation this area is mostly characterized by the degradation stage of xero-thermophyllous deciduous lower forest and thickets of oriental hornbeam (the order Ostryo-Carpinetalia orientalis Lakušić et al. 1982) of the Rusco aculeati-Carpinetum orientalis Blečić et Lakušić 1966 association (LaKušić et al., 1982; MuRAtspahić at al., 1991; Redžıć et al., 1992).

The flora of the town of Blagaj has been poorly investigated. Some published data exist, but there are no published complete lists or analyses of the flora of the area. The majority of the information was given by Struschka in the $19^{\text {th }}$ century (STRUSCHKA, 1880), in the work Die Umgebung Mostars, listing records of 48 taxa of vascular flora. There are a few other works that list individual findings of plant taxa from this area (FIALA, 1890, one taxon; Murbeck, 1891, one taxon; MALY, 1905, 1920, 1923, 1928, nine taxa; Šilić, 1972/1973, two taxa). In the Flora of Bosnia and Herzegovina (BECK, 1903-1927; BECK \& MALy, 1950; Bеск et al., 1967, 1974, 1983) a total of 85 taxa are listed for the Blagaj area (excluding the above-mentioned authors).

In the last five decades numerous papers have been published about the flora and vegetation of European towns (Sukopp; 1990, 2002; Py̌̌eK, 1998; Kelcey \& Müller, 2011). Investigations in the southern parts of Europe have been intensified in the last two decades (for references see Milović \& Mitić, 2012). The urban flora of Bosnia and Herzegovina remains almost unexplored, for only the cities of Sarajevo and Mostar have been studied (Tomović-Hadžiavdić \& Šoljan, 2006: JAsprica et al., 2011; Maslo, 2014).

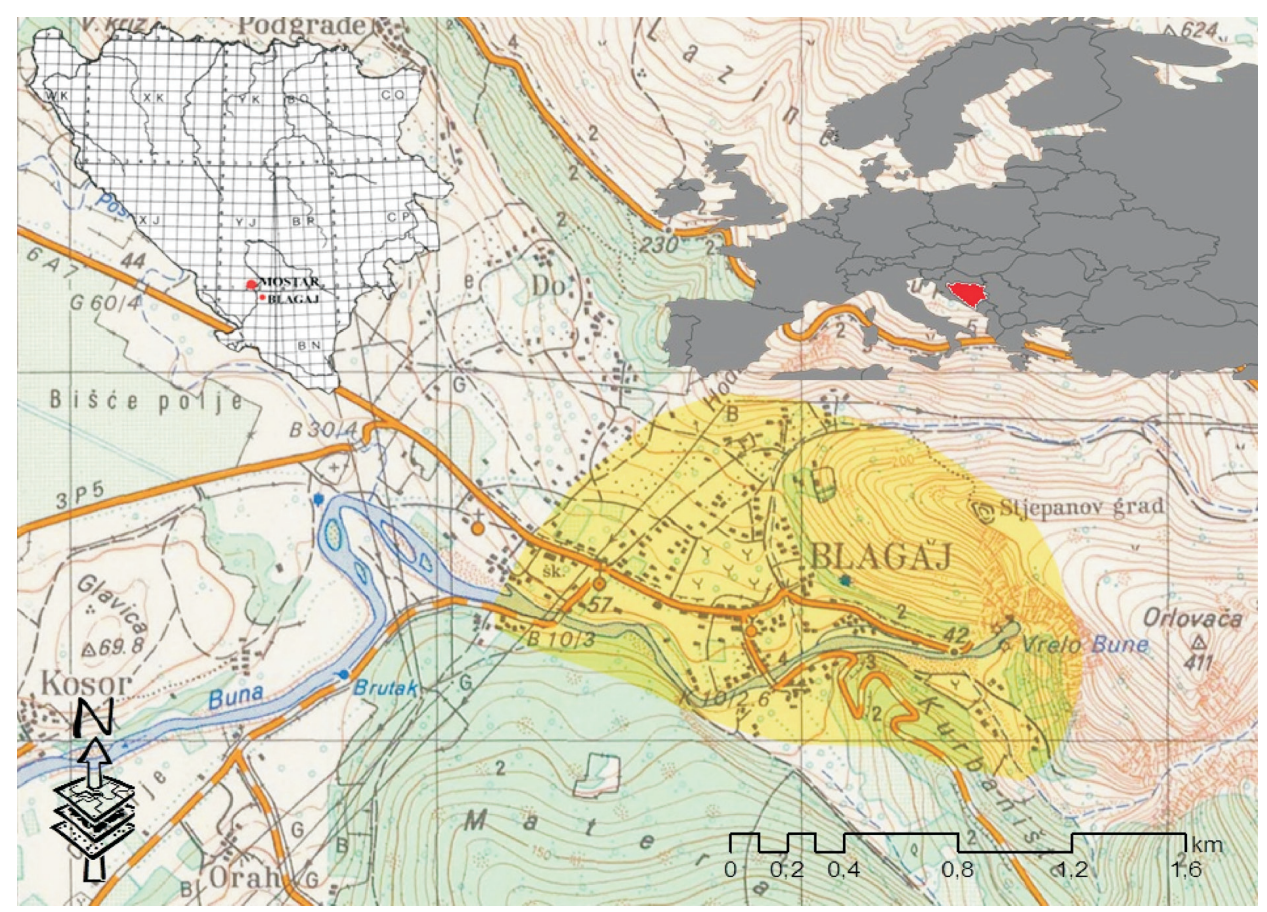

Fig.1. Location and boundaries of the researched area 
According to all acquired literature data (see References), and our own field observations, the preliminary list of vascular flora of Blagaj consists of 723 taxa. Voucher material is deposited in the Herbarium of the National Museum of Bosnia and Herzegovina (SARA).

\section{MATERIALS AND METHODS}

This study is based on all acquired literature data and the authors' field investigations from the spring of 2004 to the end of 2014. Plant nomenclature follows Nikolić (2014) and the taxa not listed there are accepted after Tutin et al. (1968-1980, 1993). In this work the Asteraceae family is perceived in a broader sense (sensu lato).

In the list of alien flora (Appendix 1), taxa were listed in alphabetic order. Designations for: family, life form, floral element, endemic/threatened status, invasion status, history, origin and first records are provided. The life-form categories follow RAUNKIAER (1934), Pignatti (1982), Diklić (1984) and are marked with the standard abbreviations in the list of flora: $\mathbf{C h}$ (Chamaephytes), G (Geophytes), H (Hemmicriptophytes), Hy (Hydrophytes), $\mathbf{P}$ (Phanerophytes) and $\mathbf{T}$ (Therophytes). The analysis of floral elements was made according to Horvatić (1963) and Horvatić et al. (1976/1968). The data about plant taxa that could not be classified according to above mentioned source were taken from Horvatić (1967), Josifović et al. (1970-1977) and Gajić (1984).

\section{MEDITERRANEAN FLORAL ELEMENT}

1.1. Circum-Mediterranean plants (CIME)

1.2. West-Mediterranean plants (WEME)

1.3. East-Mediterranean plants (EAME)

1.4. Illyrian Mediterranean plants

1.4.1. Illyrian-South European plants (ILSE)

1.4.2. Illyrian-Adriatic plants

a) Illyrian-Adriatic endemic plants (ILAE)

b) Illyrian-Apennine plants (ILAP)

1.5. Mediterranean-Atlantic plants (MEAT)

1.6. European Mediterranean plants (EUME)

1.7. Mediterranean-Pontic plants (MEPO)

2. ILLYRIAN-BALKANIC FLORAL ELEMENT

2.1. Illyrian-Balkanic endemic plants (ILBE)

2.2. Balkanic-Apennine plants (BAAP)

3. SOUTH EUROPEAN FLORAL ELEMENT

3.1. South European-Mediterranean plants (SEME)

3.2. South European-Pontic plants (SEPO)

3.3. South European-mountain plants (SEMO)

3.4. South European-continental plants (SECO)

3.5. South European-Atlantic plants (SEAT)

4. EAST EUROPEAN-PONTIC FLORAL ELEMENT (EEUP)

5. SOUTHEAST EUROPEAN FLORAL ELEMENT (SEEU)

6. CENTRAL EUROPEAN FLORAL ELEMENT (CEEU) 


\section{EUROPEAN FLORAL ELEMENT (EURO)}

8. EURASIAN FLORAL ELEMENT (EUAS)

9. CIRCUM-HOLARTIC PLANTS (CIHO)

10. WIDESPREAD PLANTS (WISP)

\section{ALIEN PLANTS (CUAD)}

Data about the geographic origin of alien taxa were taken mostly from the available literature (see References). The terminology presented below has been adapted from Richardson et al. (2000), PyšEk et al. (2004), Boršić et al. (2008) and Mitić et al. (2008). All taxa were classified into three categories depending on the degree of their naturalization: casual taxa (CAS.), naturalized non-invasive taxa (NAT.) and naturalized invasive taxa (INV.), and archaeophytes (arc.) and neophytes (neo.) with respect to residence time.

- ALIEN (NON-NATIVE, NON-INDIGENOUS, INTRODUCED) PLANTS. Plant taxa in a given area whose presence there is due to intentional or accidental introduction as a result of human activity.

- CASUAL (TRANSIENT, EPHEMERAL) PLANTS. Alien plants that may flourish and even reproduce occasionally in an area, but which do not form self-replacing populations, and which rely on repeated introductions for their persistence.

- NATURALIZED PLANTS. Alien plants that reproduce consistently and sustain populations over many life cycles without direct intervention by humans; they often recruit offspring freely, usually close to adult plants, and do not necessarily invade natural, seminatural or human-made ecosystems.

- INVASIVE PLANTS (PLANT INVADERS). Naturalized plants that produce reproductive offspring, often in very large numbers, at considerable distances from parent plants, and thus have the potential to spread over a large area.

- archaeophytes, established non-native plants introduced intentionally or unintentionally before 1500 A.D.

- neophytes, non-native plants introduced intentionally or unintentionally after 1500 A.D.

Endemic species in a broader sense are defined according to BJELčić (1987), ŠILIĆ (1990) and LubARda et al. (2014), and are marked with the abbreviation "end". Taxa listed in the proposal for the Red Book of the Flora of Bosnia and Herzegovina (ŠILIĆ, 1996) and in the Red List of Flora in the Federation of B \& H (Đug et al., 2013) are marked with their corresponding IUCN category: Extinct (EX), Extinct in the Wild (EW), Critically Endangered (CR), Endangered (EN), Vulnerable (VU), Near Threatened (NT), Least Concern (LC), Data Deficient (DD) and Not Evaluated (NE).

\section{RESULTS}

The list of the flora of Blagaj contains a total of 723 taxa, which is $15.82 \%$ of the complete flora of Bosnia and Herzegovina (4569 taxa). They are sorted into 407 genera and 102 families. Pterydophyta contribute 9 taxa. Gymnospermae are represented by only four taxa. Angiospermae are represented by 710 taxa, with a distinct domination of Dicotyledones (582 taxa, $81.97 \%$ ) over Monocotyledones (128 taxa, 18.03\%), see Tab. 1. 
Tab.1. Taxonomic analysis

\begin{tabular}{|l|c|c|c|c|c|}
\hline \multirow{2}{*}{ TAXA } & Pterydophyta & Gymnospermae & \multicolumn{2}{|c|}{ Angiospermae } & \multirow{2}{*}{ Total } \\
\cline { 2 - 5 } & & & Dicotyledones & Monocotyledones & \\
\hline Families & 5 & 2 & 79 & 16 & 102 \\
\hline Genera & 6 & 3 & 325 & 73 & 407 \\
\hline $\begin{array}{l}\text { Species \& } \\
\text { subspecies }\end{array}$ & 9 & 4 & 582 & 128 & 723 \\
\hline$\%$ & 1.24 & 0.55 & 80.51 & 17.70 & 100 \\
\hline
\end{tabular}

According to the number of taxa, the most abundant family is Asteraceae s. $l$. with 85 taxa $(11.76 \%)$, followed by Poaceae (71 taxa, 9.82\%), see Tab. 2. The most abundant genera are Trifolium (13), Euphorbia (10), Geranium (10) and Medicago (9 taxa).

Tab.2. The most abundant families in the flora of Blagaj

\begin{tabular}{|l|c|c|}
\hline Families & No.taxa & \% of total flora (716) \\
\hline Asteraceae s. $l$. & 85 & 11.76 \\
\hline Poaceae & 71 & 9.82 \\
\hline Fabaceae & 56 & 7.75 \\
\hline Lamiaceae & 47 & 6.50 \\
\hline Brassicaceae & 39 & 5.39 \\
\hline Scrophulariaceae & 30 & 4.15 \\
\hline Apiaceae & 28 & 3.87 \\
\hline
\end{tabular}

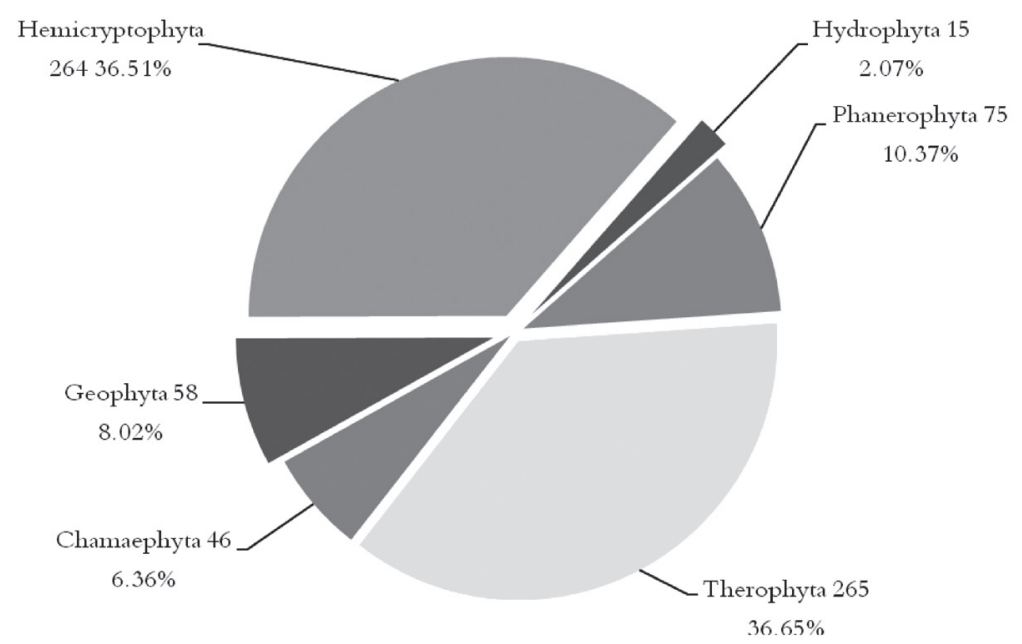

Fig.2. Life-form spectrum of flora of Blagaj 
Analysis of life-forms of the flora of Blagaj (Fig. 2.) shows the domination of therophytes and hemicryptophytes with 265 and 264 taxa, respectively (36.65\% and $36.51 \%)$, while the least abundant are hydrophytes with only 15 taxa $(2.07 \%)$.

In the spectrum of floral elements (Fig. 3), Mediterranean plants dominate (213 taxa; $29.46 \%$ ), followed by South European plants (133 taxa; $18.40 \%$ ), reflecting the phytogeographic location of the researched area.The significant numbers of alien plants (87 taxa; $12.03 \%$ ) as well as widespread plants (117 taxa; $16.18 \%$ ) are the consequence of a strong, long-lasting human influence upon the flora and the vegetation of the Blagaj region.

Alien species contributed $12.03 \%$ to the total species number, the corresponding figure being $6.36 \%$ for neophytes and $5.67 \%$ for archeophytes.

A total of 26 taxa (3.60\% of total flora) have the status of endemic plants and 38 taxa (5.26\%) are considered to be threatened (ŠILIĆ, 1996).

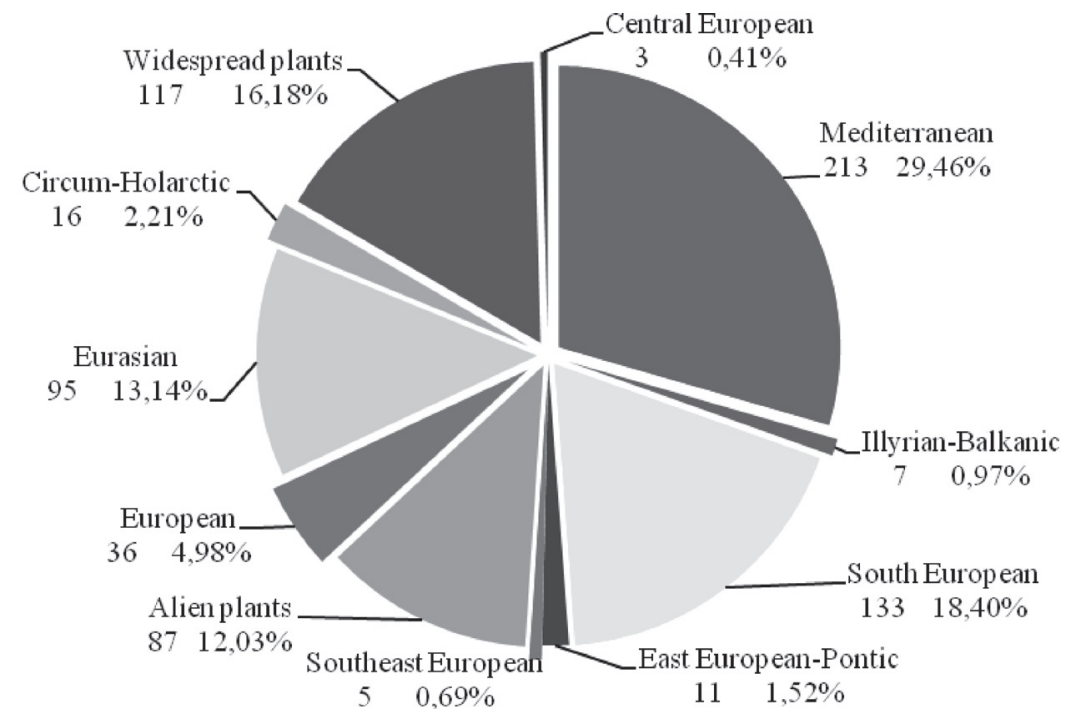

Fig.3. Spectrum of floral elements of the flora of Blagaj

According to the degree of naturalization the most prominent are casual plants with 36 taxa (41.38\%), followed by invasive plants with 27 taxa (31.03\%). There were 24 taxa $(27.59 \%)$ of naturalized plants. Analysis of the geographical origin of the alien flora of Blagaj showed that most plants originated from the Americas (34 taxa, 39.08\%), most of them from North America (18 taxa). According to the scheme proposed by RicHARDson et al., (2000) and PYš́x et al. (2004), 27 taxa of alien flora of Blagaj can be classified as invasive (Appendix 1)

\section{DISCUSSION}

In the area researched a total of 723 vascular plant taxa were recorded (Tab.1). The great richness of the flora of the relatively small area of the Blagaj region researched (4 $\mathrm{km}^{2}$ app.) results from the phytogeographical location of the town, a diversity of habitats and long-lasting anthropogenic influences. 

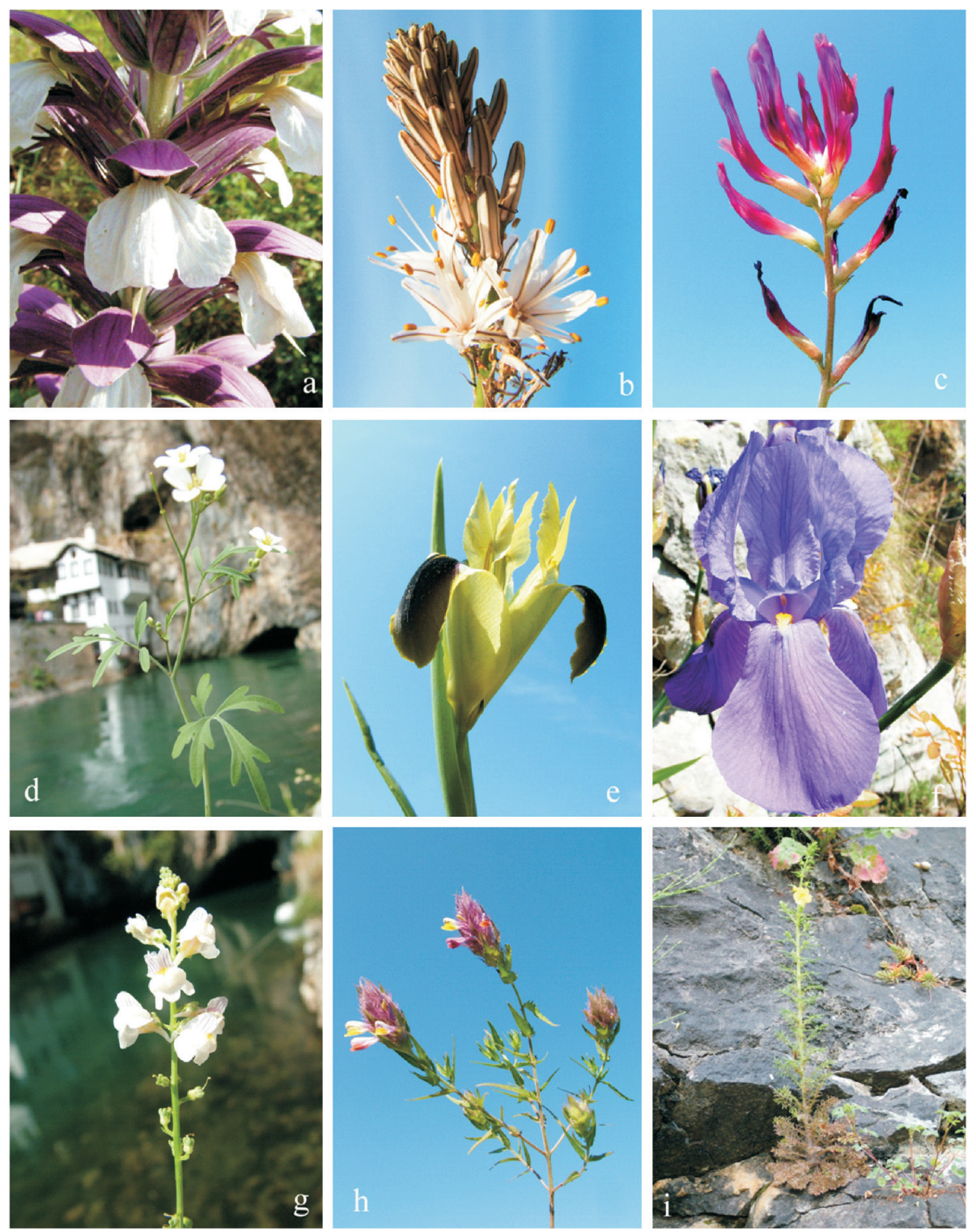

Fig.4. Some threatened taxa in the flora of Blagaj: a. Acanthus spinosissimus Pers. b. Asphodelus aestivus Brot. c. Astragalus monspessulanus L. ssp. illyricus (Bernhardt) Chater d. Cardamine maritima Port. ex DC. e. Hermodactylus tuberosus (L.) Mill. f. Iris pseudopallida Trinajstić g. Linaria microsepala A.Kern. h. Melampyrum fimbriatum Vandas i. Verbascum orientale (L.) All. (Photo: Semir Maslo).

The total number of taxa recorded for the town of Blagaj is largely similar to the numbers of taxa recorded for the city of Mostar, some Dalmatian cities and Montenegro (Tab.3). 
Tab. 3. Comparision of number of taxa among the different cities.

\begin{tabular}{|l|c|c|c|l|}
\hline City & No. of taxa & Area $\left(\mathrm{km}^{2}\right)$ & No. of inhabitants & Literature \\
\hline Blagaj (B \& H) & 723 & 4 & 2.700 & This study \\
\hline Mostar (B \& H) & 965 & 20 & 80.000 & MAsLo (2014) \\
\hline Omiš (Croatia) & 614 & 3.5 & 6.400 & TAFRA et al. (2012) \\
\hline Podgorica (Montenegro) & 1227 & 86 & 140.000 & STEŠEvić \& JovANOvić (2008) \\
\hline Split (Croatia) & 842 & 30 & 175.000 & Ruščı́ (2002) \\
\hline Šibenik (Croatia) & 617 & 4 & 40.000 & MiLović (2000) \\
\hline Zadar (Croatia) & 926 & 30 & 70.000 & MiLović \& Mitić (2012) \\
\hline
\end{tabular}

Of the 723 taxa in the flora of Blagaj, 146 taxa were previously registered and 577 taxa were recorded in the area researched for the first time in this study. Some of the listed taxa are recognized as regionally threatened (IUCN, 2001) and should be included in the Preliminary Red List of Threatened Plant Species of Bosnia and Herzegovina (ŠILIĆ, 1996).

Out of 146 taxa previously registered for the Blagaj area, 14 taxa recorded mostly by StruschKa (1880) were not confirmed by this research (Arbutus unedo, Asperula cynanchica, Crepis pannonica, Cruciata pedemontana, Euphorbia esula, Goniolimon dalmaticum, Heliotropium supinum, Hyoscyamus albus, Plantago argentea, Prunella grandiflora, Smilax aspera, Trifolium alpestre, Trifolium setiferum and Verbascum sinuatum). Some of the unconfirmed species probably do exist on the researched area but were overlooked. Others disappeared during the last 130 years probably because of urbanization and anthropogenic impact.

The most abundant plant family is Asteraceae s.1. (85 taxa; $11.76 \%$ ), followed by Poaceae (71 taxa, 9.82\%) and Fabaceae (56 taxa; 7.75\%). The same three families were dominant in the taxa in the floras of the city of Mostar (MAsLo, 2014), some Dalmatian cities: Šibenik (Milović, 2000), Split (Ruščić, 2002), Zadar (Milović \& Mitić, 2012), Omiš (Tafra et al., 2012) as well as in the flora of the city of Podgorica, Montenegro (STEŠEvić \& Jovanović, 2008).

Tab. 4. Plant life-form of the flora of Blagaj and floras of the city of Mostar (MAsLo, 2014) and some Dalmatian cities: Šibenik (Milović, 2000), Split (Ruščić, 2002), Zadar (Milović \& Mitić, 2012), Omiš (TAFra et al., 2012)

\begin{tabular}{|l|l|l|l|l|l|l|}
\hline City & Terophyta & $\begin{array}{l}\text { Hemicryp- } \\
\text { tophyta }\end{array}$ & $\begin{array}{l}\text { Phanero- } \\
\text { phyta }\end{array}$ & Geophyta & $\begin{array}{l}\text { Chamae- } \\
\text { phyta }\end{array}$ & $\begin{array}{l}\text { Hydro- } \\
\text { phyta }\end{array}$ \\
\hline Blagaj & $36.65 \%$ & $36.51 \%$ & $10.37 \%$ & $8.02 \%$ & $6.36 \%$ & $2.07 \%$ \\
\hline Mostar & $38.86 \%$ & $33.68 \%$ & $9.64 \%$ & $10.16 \%$ & $6.22 \%$ & $1.45 \%$ \\
\hline Split & $37.8 \%$ & $29.6 \%$ & $15.6 \%$ & $9.5 \%$ & $6.7 \%$ & - \\
\hline Šibenik & $47.65 \%$ & $27.55 \%$ & $10.7 \%$ & $6.81 \%$ & $7.29 \%$ & - \\
\hline Zadar & $42.98 \%$ & $26.57 \%$ & $12.85 \%$ & $11.02 \%$ & $6.26 \%$ & $0.32 \%$ \\
\hline Omiš & $40.07 \%$ & $28.34 \%$ & $14.82 \%$ & $7.98 \%$ & $8.63 \%$ & $0.16 \%$ \\
\hline
\end{tabular}


Therophytes and hemicryptophytes account for the highest number of life forms in the flora of Blagaj with 265 and 264 taxa, respectively (36.65\% and 36.51\%), while hydrophytes are the least abundant with only 15 taxa (2.07\%).The given results match the data for the city of Mostar and those for some Dalmatian cities (Tab.4). The dominance of therophytes in urban floras is not unexpected as their short life-cycles and high numbers of easily dispersed seeds make these plants very effective colonizers of heterogeneous habitats.

The comparison of floral elements of the flora of Blagaj with the floras of the city of Mostar and those for some Dalmatian cities (Tab.5) shows that there are great similarities in the highest presence of Mediterranean taxa and a significant presence of South European, widespread and alien taxa, as well. These data indicate that the floral assemblages (in terms of life-forms and floral elements) of Mediterranean urban areas mainly result from the general conditions of the Mediterranean climate as well as from anthropogenic impacts.

Tab. 5. Comparison of the floral elements among the different cities.

\begin{tabular}{|l|l|l|l|l|l|l|}
\hline $\begin{array}{l}\text { Floral } \\
\text { element }\end{array}$ & Blagaj & Mostar & Split & Šibenik & Zadar & Omiš \\
\hline $\begin{array}{l}\text { Mediterra- } \\
\text { nean }\end{array}$ & $29.46 \%$ & $26.94 \%$ & $36.2 \%$ & $39.71 \%$ & $32.83 \%$ & $37.95 \%$ \\
\hline $\begin{array}{l}\text { South } \\
\text { European }\end{array}$ & $18.40 \%$ & $18.65 \%$ & $16.70 \%$ & $19.94 \%$ & $17.06 \%$ & $16.45 \%$ \\
\hline Eurasian & $13.14 \%$ & $14.61 \%$ & $8.80 \%$ & $7.46 \%$ & $9.61 \%$ & $7.82 \%$ \\
\hline $\begin{array}{l}\text { Widespread } \\
\text { plants }\end{array}$ & $16.18 \%$ & $13.47 \%$ & $15.80 \%$ & $17.18 \%$ & $15.55 \%$ & $16.78 \%$ \\
\hline $\begin{array}{l}\text { Alien } \\
\text { plants }\end{array}$ & $12.03 \%$ & $15.65 \%$ & $16.60 \%$ & $10.53 \%$ & $19.22 \%$ & $17.43 \%$ \\
\hline Others & $10.79 \%$ & $10.68 \%$ & $5.90 \%$ & $5.18 \%$ & $5.73 \%$ & $3.57 \%$ \\
\hline Total & $100 \%$ & $100 \%$ & $100 \%$ & $100 \%$ & $100 \%$ & $100 \%$ \\
\hline
\end{tabular}

\section{CONCLUSIONS}

In the total of 723 plant taxa that were identified in this research, 577 of them were listed for the first time for the flora of the Blagaj. The findings of four taxa from the area are of special interest because they were not previously reported for the flora of Bosnia and Herzegovina (Fumaria gaillardotii, Orobanche hederae, Saccharum strictum and Theligonum cynocrambe).

Some other taxa were recorded only here, or in one or just a few localities in Bosnia and Herzegovina (Ammi majus, Aristolochia rotunda, Arum nigrum, Asphodelus aestivus, Asphodelus fistulosus, Carex distachya, Cerinthe minor ssp. auriculata, Cheilanthes persica, Clypeola jonthlaspi, Crepis pannonica, Erysimum linariifolium, Goniolimon dalmaticum, Hedypnois cretica, Hermodactylus tuberosus, Hyoscyamus albus, Iris pseudopallida, Lavatera cretica, Legousia hybrida, Linaria microsepala, Melampyrum fimbriatum, Opopanax chironium, Phleum echinatum, Piptatherum holciforme, Stenbergia lutea and Verbascum orientale). 

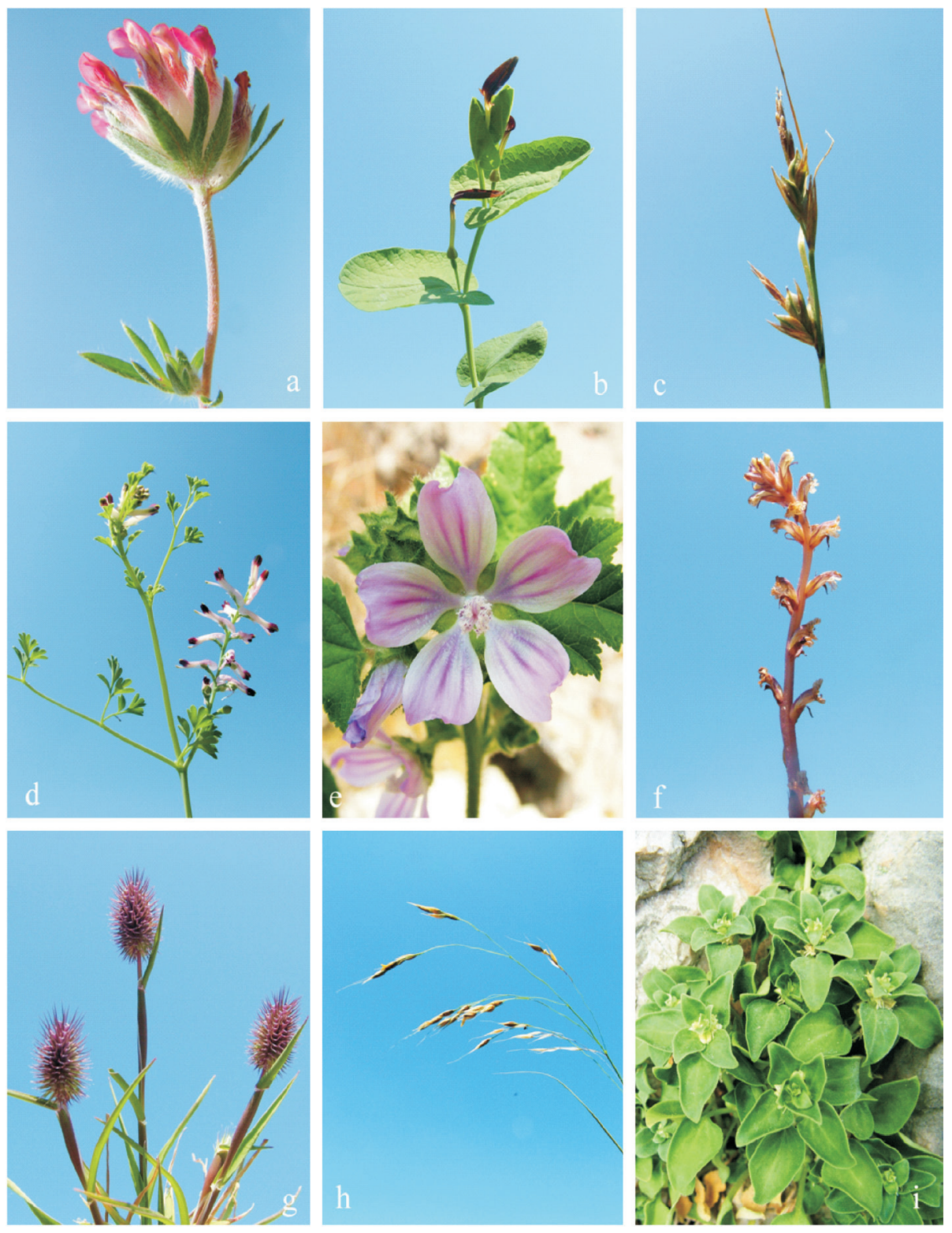

Fig.5. Some rare taxa in the flora of Blagaj: a. Anthyllis vulneraria L. ssp. praepropera Bornm. B. Aristolochia rotunda L. C. Carex distachya Desf. d. Fumaria gaillardotii Boiss. E. Lavatera cretica L. F. Orobanche hederae Duby. G. Phleum echinatum Host. H. Piptatherum holciforme (M.Bieb.) Roem. \& Schult. I. Theligonum cynocrambe L. (Photo: Semir Maslo).

According to the Red List of Flora in the Federation of Bosnia and Herzegovina (ĐUG et al. 2013), 38 taxa of the flora of the town of Blagaj are in some threat category. Six taxa $(15.79 \%)$ are categorized as Critically Endangered (CR), seven taxa (18.42\%) as Endan- 
gered (EN), six taxa (15.79\%) as Vulnerable (VU), six taxa (15.79\%) as Near Threatened (NT), 3 taxa (7.89\%) as Least Concern (LC) and ten taxa (26.32\%) as Data Deficient (DD).

Our study has confirmed that the floras of urban areas are extremely rich and diverse. The greater diversity of the flora of Blagaj is attributable both to geographic and cultural factors, and to the marked presence of Mediterranean species which, favoured by the urban climate, have successfully settled in this environment.

We found that the vascular flora of Blagaj is very close to the Mediterranean flora. The vascular flora of Blagaj showed many common characteristics with floras of Mostar and some Dalmatian cities, both qualitatively and quantitatively. It seems that the Blagaj area is more exposed to the Mediterranean climate than Mostar. This is supported by the presence of typical Mediterranean species that do not grow in the Mostar area, and are found only in the southern Herzegovina or in neighbouring Dalmatia: Asphodelus aestivus, Fumaria gaillardotii, Goniolimon dalmaticum, Hedypnois cretica, Hyoscyamus albus, Iris pseudopallida, Saccharum strictum and Theligonum cynocrambe.This is probably related to the physiographic position of Blagaj area which is wide open to the southwest and closed to the northeast by the high cliffs of Orlovača Hill.

In summary, the vascular flora of Blagaj can be considered to be vascular flora typical of sub-Mediterranean cities.

Received September 7, 2014

\section{REFERENCES}

Beck-Mannagetta, G., 1903: Flora Bosne, Hercegovine i Novopazarskog Sandžaka, Gymnospermae i Monocotyledones, I dio Državna štamparija, Sarajevo.

Beck-Mannagetta, G., 1916: Flora Bosne, Hercegovine i Novopazarskog Sandžaka, II , Sarajevo.

Beck-Mannagetta, G., 1927: Flora Bosne i Hercegovine i oblasti Novog Pazara, III -Horipetalae. Beograd - Sarajevo.

Beck, G. M. \& Maly, K., 1950: Flora Bosnae et Hercegovinae, IV Sympetalae, pars I, 1-72, Svjetlost, Sarajevo.

Beck, G., Maly, K. \& BJelčıć, Ž., 1967: Flora Bosnae et Hercegovinae, IV Sympetalae, 2, Zemaljski muzej $\mathrm{BiH}$, Sarajevo.

BecK, G., MALY, K. \& BJELČIĆ, Ž., 1974: Flora Bosne i Hercegovine IV - Sympetalae 3. Sarajevo.

Beck, G., MALY, K. \& BJElČIĆ, Ž., 1983: Flora Bosne i Hercegovine IV - Sympetalae 4. Sarajevo.

BJELČIĆ, Ž., 1987: Endemi u biljnom svijetu Bosne i Hercegovine i problemi zaštite, ANU BiH Sarajevo.

Boršić, I., Milović, M., Dujmović, I., Bogdanović, S., Cigić, P., RešEtnik, I., Nikolić, T. \& Mitić, B., 2008 : Preliminary check-list of invasive alien plant species (IAS) in Croatia. Nat.Croat., Vol. 17(2).

DIKLIĆ, N., 1984: Životne forme biljnih vrsta i biološki spektar flore SR Srbije. In SARIć, M. (ed.), Vegetacija SR Srbije I, 291-316. SANU, Beograd.

Đug, S., Muratović, E., Drešković, N., Boškailo, A. \& Dudević, S., 2013: Crvena lista flore Federacije BiH, knjiga 2, Federalno ministarstvo okoliša i turizma.

Fiala, F., 1890: Prilozi flori Bosne i Hercegovine. Glas. Zem. Muz. Bosne. Herceg. 2, 309-315.

GajIĆ, M., 1984: Florni elementi SR Srbije. In SARIć, M. (ed.), Vegetacija SR Srbije I, 317-397. SANU, Beograd.

Horvatić, S., 1963: Vegetacijska karta otoka Paga s općim pregledom vegetacijskih jedinica Hrvatskog primorja. JAZU, Odjel za prirodne nauke, Zagreb.

HoRvatić, S., 1967: Analitička flora Jugoslavije 1(1). Institut za Botaniku Sveučilista u Zagrebu, Grafički zavod Hrvatske, Zagreb.

Horvatić, S., Ilijanić, I. \& Marković-Gospodarić, 1967/1968: Biljni pokrov okoline Senja. Senjski zbornik, 298-321. 
Hrvatović, H., 2006: Geological Guidebook through Bosnia and Herzegovina, Federal Institute for Geology, Sarajevo.

IUCN, 2001: IUCN Red List Categories and Criteria: Version 3.1. IUCN Species Survival Commission. Gland, Cambridge.

Jasprica, N., Ruščić. M., Lasić, A., 2011: Comparison of urban flora in Dubrovnik, Split and Mostar. Hrvatska misao (Matica hrvatska Sarajevo). 55 (3), nova serija sv. 40, 77-104.

Josifović, M. (ed.), 1970-1977: Flora SR Srbije, I-IX. SANU, Beograd.

Kelcey, J.G. \& Müller, N. (eds.), 2011: Plants and Habitats of European cities, Verlag Springer, New York.

LAKUŠić, R., PAVlović, D., \& S. Redžı́c, 1982: Horološko-ekološka i floristička diferencijacija šuma i šikara sa bjelograbićem (Carpinus orientalis Mill.) i crnim grabom (Ostrya carpinifolia Scop.) na prostoru Jugoslavije. Glas. Republ. Zavoda zašt. prirode - Prirodnjačkog muzeja, 15, 103-116.

Lubarda, B., Stupar, V., Milanović, Đ. \& Stevanović V., 2014: Chorological characterization and distribution of the Balkan endemic vascular flora in Bosnia and Herzegovina. Botanica Serbica. 38 (1), 167-184.

MALY, K., 1905: Izvještaj o izletu članova međunarodnog botaničkog kongresa u Beču u Bosnu, godine 1905, Glas. Zem. Muz. Bosne. Herceg. 17, 483-487.

Maly, K., 1920: Prilozi za floru Bosne i Hercegovine 7\&8. Glas. Zem. Muz. Bosne. Herceg. 32, 129-139.

MaLy, K., 1923: Prilozi za floru Bosne i Hercegovine 9. Glas. Zem. Muz. Bosne. Herceg. 35, 123-162.

MaLy, K., 1928: Prilozi za floru Bosne i Hercegovine 10. Glas. Zem. Muz. Bosne. Herceg. 40, 107-166.

MAsLo, S., 2014: The urban flora of the city of Mostar (Bosnia and Herzegovina). Nat. Croat. 23(1), 65-109.

Mılović, M., 2000: Flora papratnjača i sjemenjača Šibenika i okolice. MSc Thesis. Faculty of Science. University of Zagreb, Zagreb.

Milović, M. \& Mitrć, B, 2012: The urban flora of the city of Zadar (Dalmacija, Croatia). Nat. Croat. 21(1), $65-100$.

Mitić, B., Boršić, I., Dujmović, I., Bogdanović, S., Milović, M., Cigić, P., ReŠEtnik, I. \& Nikolić, T., 2008: Alien flora of Croatia: proposals for standards in terminology, criteria and related database. Nat. Croat. 17, 73-90.

Muratspahić, D., Redžıć, S. \& R. LaKušrć, 1991: Asocijacija Rusco -Carpinetum orientalis Bleč. et Lkšić 1966 u dolini rijeke Neretve. Glas. Republ. zavoda zašt. Prirode - Prirodnjačkog muzeja, 24, 7-12.

Murbeck, S., 1891: Beitrage zur Kenntnis der Flora von Sudbosnien und der Hercegovina. Lunds Universitets Arsskrift, 27: 1-182, Lund.

NiкоLı́́, T. (ed.), 2014: Flora Croatica baza podataka / Flora croatica Database. On-Line URL: http://hirc. botanic.hr/fcd. (Accessed April 2014). Botanički zavod, Prirodoslovno-matematički fakultet, Sveučilište u Zagrebu.

PignatTi, S. (ed.), 1982: Flora D'Italia 1-3. Edagricole, Bologna.

PYšEK, P., 1998: Alien and native species in Central European urban floras: a quantitative comparsion. J. Biogeogr. 25, 155-163.

Pyšek, P., Richardson, D.M., Rejmanek, M., Webster, G.L., Williamson, M., \& Kirschner, J., 2004: Alien plants in checklists and floras: towards better communication between taxonomists and ecologists. Taxon, 53(1), 131-143.

Raunkiaer, C., 1934: The life forms of plants and statical plant geography. Clarendon Press, Oxford.

Redžić, S., Muratspahić, D., LaKušić, R., 1992: Neke fitocenoze šuma i šikara iz doline Neretve. Poljoprivreda i šumarstvo 38 (1-2), 95-101.

Richardson, D. M., Pyšek P., Rejmánek M., Barbour M. G., Panetta F. D. \& West C. J., 2000: Naturalization and invasion of alien plants: concepts and definitions. Diversity \& Distributions, Oxford, 6, 93107.

Rivas-Martínez, S., Penas, A., Diasz, T.E., 2004: Biogeographic Map of Europé. Cartographic Service. University of León, Spain.

Ruščić, M., 2002: Urbana flora grada Splita. MSc Thesis. Faculty of Science. University of Zagreb, Zagreb.

Stešević, D. \& Jovanović, S., 2008: Flora of the city of Podgorica, Montenegro-Taxonomic analysis. Arch. Biol.Sci. Belgrade 60(2), 245-253.

SteŠEvić, D., Jovanović, S. \& S. ŠćEPanović, 2009: Flora of the city of Podgorica, Montenegro -Chorological structure and comparison with the floras of Rome, Patras and Salonika. Arch.Biol.Sci. Belgrade 61(2), 307-315.

StruschKa, H., 1880: Die umgebung Mostars. (Jahresb. k. k. Staats-Gymnas., 1880.) 44 pp. Kremsier. 
Šılıć, Č., 1972/1973: Nova nalazišta nekih rijetkih i manje poznatih biljnih vrsta u flori Bosne i Hercegovine. Glasnik Zemaljskog muzeja Bosne i Hercegovine u Sarajevu, 11-12, 59-79.

ŠILIć, Č., 1973: Tagetes minutus L. - sve masovniji i sve opasniji korov na poloprivrednim površinama Dalmacije, Hercegovine, Crnogorskog primorja i južne Makedonije. Jugoslovenski simpozijum o borbi protiv korova u brdsko-planinskim područjima, 27-34.

ŠILIĆ, Č., 1990: Endemične biljke. III izdanje, IP „Svjetlost“; Zavod za udžbenike i nastavna sredstva, Sarajevo; Zavod za udžbenike i nastavna sredstva, Beograd.

ŠILrć, Č., 1996: Spisak biljnih vrsta (Pteridophyta i Spermatophyta) za Crvenu knjigu Bosne i Hercegovine. Glas. Zem. Muz. Bosne. Herceg. (PN) (NS), sv. 31, 323-367.

Suкорт, H., 1990: Urban ecology and its application in Europe. In: Suкоpr, H.\& S. HejnY, (eds.), Kowarnik, I. (co-ed.): Urban Ecology. Plants and plant communities in urban environments. SPB Academic Publishing, The Hague, 1-21.

Suкорт, H., 2002: On the early history of urban ecology in Europe. Preslia 74, 373-393.

Tomović-HadžIAvDić, V. \& ŠoljAN, D., 2006: Urbana flora Sarajeva. /Urban Flora of Sarajevo./ GZM (PN) NS 32, 121-135.

TAfra, D., PAndžA, M., Milović, M., 2012: Vascular flora of the town of Omiš. Nat. Croat. 21(2), 301-334.

Tutin, T.G., Burges, N. A., Chater, A. O., Edmondson, J. R., Heywood, V. H., Moore, D. M., Valentine, D. H., Walters, S. M. \& D. A. Webb, (eds.), 1993: Flora Europea 1, 2nd ed. Cambridge University Pres, Cambridge.

Tutin, T.G., Heywood, V.H., Burges, N. A., Moore, D. M., Valentine, D. H, Walters, S. \& Weeb, D. A. (eds.), 1968 - 1980: Flora Europea 2-5. Cambridge University Pres, Cambridge. 


\section{APPENDIX 1. VASCULAR FLORA OF THE TOWN OF BLAGAJ \\ (If the author of the first record is not cited, the species is quoted here for the first time)}

\begin{tabular}{|c|c|c|c|c|c|c|c|c|c|c|}
\hline \multirow[b]{2}{*}{ 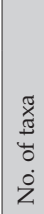 } & \multirow[b]{2}{*}{$\underset{\widetilde{\Xi}}{\widetilde{\pi}}$} & \multirow[b]{2}{*}{ 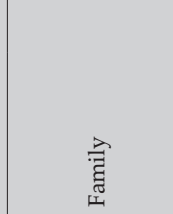 } & \multirow[b]{2}{*}{ 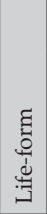 } & \multirow{2}{*}{$\begin{array}{l}0 \\
0 \\
0 \\
00 \\
\tilde{0} \\
0 \\
00 \\
0 \\
0 \\
0 \\
0 \\
0 \\
0\end{array}$} & \multirow[b]{2}{*}{ 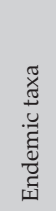 } & \multirow{2}{*}{ 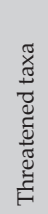 } & \multicolumn{3}{|c|}{ Alien plants (CUAD) } & \multirow{2}{*}{ 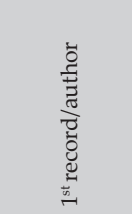 } \\
\hline & & & & & & & 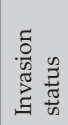 & 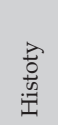 & $\begin{array}{l}.50 \\
0 \\
0\end{array}$ & \\
\hline 1. & Abutilon theophrasti Medik. & Malvaceae & $\mathrm{T}$ & CUAD & & & INV & $\operatorname{arc}$ & As-E & \\
\hline 2. & $\begin{array}{l}\text { Acanthus balcanicus Heywood et I. } \\
\text { Richardson }\end{array}$ & Acanthaceae & $\mathrm{H}$ & SEME & & & & & & BECK 1950 \\
\hline 3. & Acanthus spinosissimus Pers. & Acanthaceae & $\mathrm{H}$ & ILAP & & LC & & & & BECK 1950 \\
\hline 4. & Acer campestre $\mathrm{L}$. & Aceraceae & $\mathrm{P}$ & EURO & & & & & & \\
\hline 5. & Acer monspessulanum $\mathrm{L}$. & Aceraceae & $\mathrm{P}$ & SEME & & & & & & \\
\hline 6. & Acer negundo L. & Aceraceae & $\mathrm{P}$ & CUAD & & & INV & neo & Am-N & \\
\hline 7. & Achillea millefolium $\mathrm{L}$. & Asteraceae & $\mathrm{H}$ & WISP & & & & & & \\
\hline 8. & Acinos arvensis (Lam.) Dandy & Lamiaceae & $\mathrm{T}$ & EURO & & & & & & \\
\hline 9. & Adiantum capillus - veneris $\mathrm{L}$. & Adiantaceae & $\mathrm{H}$ & MEAT & & VU & & & & STRUSC1880 \\
\hline 10. & Adonis flammea Jacq. & Ranunculaceae & $\mathrm{T}$ & CUAD & & & NAT & $\operatorname{arc}$ & M & \\
\hline 11. & Aegilops geniculata Roth & Poaceae & $\mathrm{T}$ & CIME & & & & & & \\
\hline 12. & Aegilops triunicialis $\mathrm{L}$. & Poaceae & $\mathrm{T}$ & CIME & & & & & & \\
\hline 13. & Aethionema saxatile (L.) R.Br. & Brassicaceae & $\mathrm{Ch}$ & SEME & & & & & & \\
\hline 14. & Agrimonia eupatoria L. & Rosaceae & $\mathrm{H}$ & СIHO & & & & & & \\
\hline 15. & Agrostis stolonifera $\mathrm{L}$. & Poaceae & $\mathrm{H}$ & $\mathrm{CIHO}$ & & & & & & \\
\hline 16. & Albizia julibrissin Durazz. & Fabaceae & $\mathrm{P}$ & CUAD & & & CAS & neo & Paleo. & \\
\hline 17. & Ailanthus altisima (Mill.) Sw. & Simaroubaceae & $\mathrm{P}$ & CUAD & & & INV & neo & As-E & \\
\hline 18. & Ajuga chamaepitys (L.) Schreb. & Lamiaceae & $\mathrm{T}$ & CIME & & & & & & BECK 1950 \\
\hline 19. & Ajuga genevensis L. & Lamiaceae & $\mathrm{H}$ & EURO & & & & & & BECK 1950 \\
\hline 20. & Alcea biennis Winterl. & Malvaceae & $\mathrm{H}$ & CUAD & & & NAT & neo & M & \\
\hline 21. & Alisma plantago-aquatica $\mathrm{L}$. & Alismataceae & Hy & WISP & & & & & & \\
\hline 22. & $\begin{array}{l}\text { Alliaria petiolata (M.Bieb.) Cavara \& } \\
\text { Grande }\end{array}$ & Brassicaceae & $\mathrm{H}$ & EUAS & & & & & & \\
\hline 23. & Allium ampeloprasum $\mathrm{L}$. & Liliaceae & G & CIME & & & & & & \\
\hline 24. & Allium carinatum L. & Liliaceae & $G$ & EURO & & & & & & \\
\hline 25. & Allium cepa $\mathrm{L}$. & Liliaceae & G & CUAD & & & CAS & $\operatorname{arc}$ & As-W & \\
\hline 26. & Allium flavum L. ssp. flavum & Liliaceae & G & SEME & & & & & & \\
\hline 27. & $\begin{array}{l}\text { Allium guttatum Steven subsp. } \\
\text { dalmaticum (A.Kern. ex Janch.) } \\
\text { Stearn. }\end{array}$ & Liliaceae & G & ILBE & end & DD & & & & \\
\hline 28. & Allium roseum $\mathrm{L}$. & Liliaceae & $G$ & CIME & & & & & & \\
\hline
\end{tabular}




\begin{tabular}{|c|c|c|c|c|c|c|c|c|c|c|}
\hline 29. & $\begin{array}{l}\text { Allium sphaerocephalon L. ssp. } \\
\text { sphaerocephalon }\end{array}$ & Liliaceae & G & SEME & & & & & & BECK 1903 \\
\hline 30. & Alopecurus myosuroides Huds. & Poaceae & $\mathrm{T}$ & WISP & & & & & & \\
\hline 31. & Althaea cannabina $\mathrm{L}$. & Malvaceae & $\mathrm{H}$ & SEPO & & & & & & \\
\hline 32. & Alyssoides utriculata (L.) Medik. & Brassicaceae & $\mathrm{H}$ & SEME & & & & & & \\
\hline 33. & Alyssum alyssoides (L.) L. & Brassicaceae & $\mathrm{H}$ & SEME & & & & & & \\
\hline 34. & Alyssum hirsutum M. Bieb. & Brassicaceae & $\mathrm{T}$ & CIME & & & & & & \\
\hline 35. & Amaranthus albus L. & Amaranthaceae & $\mathrm{T}$ & CUAD & & & NAT & neo & Am-N & \\
\hline 36. & Amaranthus deflexsus L. & Amaranthaceae & $\mathrm{T}$ & CUAD & & & NAT & neo & Am-S & \\
\hline 37. & Amaranthus hybridus L. & Amaranthaceae & $\mathrm{T}$ & CUAD & & & NAT & neo & Am-N & \\
\hline 38. & Amaranthus retroflexus $\mathrm{L}$. & Amaranthaceae & $\mathrm{T}$ & CUAD & & & INV & neo & Am-N & \\
\hline 39. & Ambrosia artemisifolia $\mathrm{L}$. & Asteraceae & $\mathrm{T}$ & CUAD & & & INV & neo & Am-N & \\
\hline 40. & Ammi majus L & Apiaceae & $\mathrm{T}$ & SEME & & & & & & \\
\hline 41. & Anagallis arvensis $\mathrm{L}$. & Primulaceae & $\mathrm{T}$ & WISP & & & & & & \\
\hline 42. & Anagallis coerulea Schreb. & Primulaceae & $\mathrm{T}$ & WISP & & & & & & \\
\hline 43. & Anchusa arvensis (L.) M.Bieb. & Boraginaceae & $\mathrm{T}$ & EURO & & & & & & \\
\hline 44. & Anchusa cretica Mill. & Boraginaceae & $\mathrm{H}$ & CIME & & & & & & \\
\hline 45. & Anchusa italica Retz. & Boraginaceae & $\mathrm{H}$ & SEME & & & & & & \\
\hline 46. & Anchusa officinalis L. & Boraginaceae & $\mathrm{H}$ & EURO & & & & & & \\
\hline 47. & Anemone hortensis L. & Ranunculaceae & G & EUME & & & & & & \\
\hline 48. & Anthemis arvensis $\mathrm{L}$. & Asteraceae & $\mathrm{T}$ & CIME & & & & & & \\
\hline 49. & Anthemis segetalis Ten & Asteraceae & $\mathrm{T}$ & ILSE & & & & & & \\
\hline 50. & Anthoxanthum odoratum $\mathrm{L}$. & Poaceae & $\mathrm{T}$ & EUAS & & & & & & \\
\hline 51. & Anthriscus cerefolium (L.) Hoffm. & Apiaceae & $\mathrm{T}$ & EEUP & & & & & & \\
\hline 52. & $\begin{array}{l}\text { Anthyllis vulneraria L. ssp. } \\
\text { praepropera Bornm. }\end{array}$ & Fabaceae & $\mathrm{T}$ & ILAE & end & & & & & \\
\hline 53. & Antirrhinum majus L. & Scrophulariaceae & $\mathrm{T}$ & CUAD & & & NAT & $\operatorname{arc}$ & M & \\
\hline 54. & Arabis collina Ten. & Brassicaceae & $\mathrm{T}$ & SEME & & & & & & \\
\hline 55. & Arabis hirsuta (L.) Scop. & Brassicaceae & $\mathrm{T}$ & WISP & & & & & & \\
\hline 56. & Arabis turrita $\mathrm{L}$. & Brassicaceae & $\mathrm{H}$ & SEME & & & & & & \\
\hline 57. & Arbutus unedo L. & Ericaceae & $\mathrm{P}$ & CIME & & & & & & $\begin{array}{l}\text { STRUSC } \\
1880\end{array}$ \\
\hline 58. & Arceuthobium oxycedri (DC.) M. Bieb & Loranthaceae & $\mathrm{P}$ & SEPO & & & & & & \\
\hline 59. & Arctium lappa $\mathrm{L}$. & Asteraceae & $\mathrm{H}$ & EUAS & & & & & & \\
\hline 60. & Arctium minus Bernh. & Asteraceae & $\mathrm{H}$ & CIME & & & & & & \\
\hline 61. & Arenaria leptoclados (Reichenb.) Guss. & Caryophyllaceae & $\mathrm{T}$ & EUAS & & & & & & \\
\hline 62. & Arenaria serpyllifolia $\mathrm{L}$. & Caryophyllaceae & $\mathrm{T}$ & WISP & & & & & & \\
\hline 63. & Aristolochia clematitis L. & Aristolochiaceae & G & SEPO & & & & & & \\
\hline 64. & Aristolochia rotunda $\mathrm{L}$. & Aristolochiaceae & G & CIME & & EN & & & & \\
\hline 65. & Artemisia absinthium L. & Asteraceae & $\mathrm{Ch}$ & EUAS & & & & & & \\
\hline
\end{tabular}




\begin{tabular}{|c|c|c|c|c|c|c|c|c|c|c|}
\hline 66. & Artemisia anпua $\mathrm{L}$. & Asteraceae & $\mathrm{T}$ & CUAD & & & INV & neo & As-E & \\
\hline 67. & Artemisia vulgaris $\mathrm{L}$. & Asteraceae & $\mathrm{H}$ & WISP & & & & & & \\
\hline 68. & Arum italicum Miller & Araceae & G & MEAT & & & & & & \\
\hline 69. & Arum nigrum Schott & Araceae & G & ILBE & end & VU & & & & MALY 1905 \\
\hline 70. & Asparagus acutifolius L. & Asparagaceae & G & CIME & & & & & & BECK 1903 \\
\hline 71. & Asperula aristata L.f. & Rubiaceae & $\mathrm{H}$ & SEME & & & & & & \\
\hline 72. & Asperula cynanchica L. & Rubiaceae & $\mathrm{H}$ & SEME & & & & & & $\begin{array}{c}\text { STRUSC } \\
1880 \\
\end{array}$ \\
\hline 73. & Asphodeline liburnica (Scop.) Reich. & Liliaceae & G & ILSE & & & & & & \\
\hline 74. & Asphodeline lutea (L.) Rchb. & Liliaceae & G & EAME & & & & & & \\
\hline 75. & Asphodelus aestivus Brot. & Asparagaceae & G & CIME & & VU & & & & $\begin{array}{c}\text { STRUSC } \\
1880\end{array}$ \\
\hline 76. & Asphodelus fistulosus L. & Asparagaceae & $\mathrm{H}$ & CIME & & $\mathrm{CR}$ & & & & \\
\hline 77. & Asplenium ceterach L. ssp. ceterach & Aspleniaceae & $\mathrm{H}$ & SEME & & & & & & BECK 1903 \\
\hline 78. & Asplenium onopteris $\mathrm{L}$ & Aspleniaceae & $\mathrm{H}$ & CIME & & & & & & \\
\hline 79. & Asplenium ruta-muraria $\mathrm{L}$. & Aspleniaceae & $\mathrm{H}$ & $\mathrm{CIHO}$ & & & & & & BECK 1903 \\
\hline 80. & $\begin{array}{l}\text { Asplenium trichomanes L. ssp. } \\
\text { quadrivalens D.E.Mey. }\end{array}$ & Aspleniaceae & $\mathrm{H}$ & WISP & & & & & & BECK 1903 \\
\hline 81. & Aster amellus L. & Asteraceae & $\mathrm{H}$ & EEUP & & & & & & $\begin{array}{c}\text { STRUSC } \\
1880 \\
\end{array}$ \\
\hline 82. & Astragalus glycyphyllos L. & Fabaceae & $\mathrm{H}$ & EEUP & & & & & & BECK 1927 \\
\hline 83. & $\begin{array}{l}\text { Astragalus monspessulanus L. ssp. } \\
\text { illyricus (Bernhardt) Chater }\end{array}$ & Fabaceae & $\mathrm{H}$ & ILAE & end & NT & & & & \\
\hline 84. & Asyneuma limonifolium (L.) Janchen & Campanulaceae & $\mathrm{H}$ & ILAP & & & & & & \\
\hline 85. & Avena barbata Pott. ex Link. & Poaceae & $\mathrm{T}$ & WISP & & & & & & \\
\hline 86. & Avena sativa $\mathrm{L}$. & Poaceae & $\mathrm{T}$ & CUAD & & & CAS & $\operatorname{arc}$ & \begin{tabular}{|l|} 
Un- \\
kno.
\end{tabular} & \\
\hline 87. & Avena sterilis L. & Poaceae & $\mathrm{T}$ & SEPO & & & & & & \\
\hline 88. & $\begin{array}{l}\text { Ballota nigra L. ssp. foetida (Lam.) } \\
\text { Hay. }\end{array}$ & Lamiaceae & $\mathrm{H}$ & SEME & & & & & & \\
\hline 89. & Ballota rupestris (Biv. ) Vis. & Lamiaceae & $\mathrm{Ch}$ & SEMO & & & & & & \\
\hline 90. & Bellis perennis L. & Asteraceae & $\mathrm{H}$ & EURO & & & & & & \\
\hline 91. & Berteroa mutabilis (Vent.) DC. & Brassicaceae & $\mathrm{H}$ & EAME & & & & & & \\
\hline 92. & Berula erecta (Hudson) Coville & Apiaceae & G & $\mathrm{CIHO}$ & & & & & & \\
\hline 93. & Betonica officinalis L. ssp. officinalis & Lamiaceae & $\mathrm{H}$ & EURO & & & & & & BECK 1974 \\
\hline 94. & Bidens subalternans DC. & Asteraceae & $\mathrm{T}$ & CUAD & & & INV & neo & Am-S & \\
\hline 95. & Biscutella cichoriifolia Loisel. & Brassicaceae & $\mathrm{T}$ & SEME & & & & & & \\
\hline 96. & $\begin{array}{l}\text { Brachypodium sylvaticum (Huds.) P. } \\
\text { Beauv. }\end{array}$ & Poaceae & $\mathrm{H}$ & EUAS & & & & & & \\
\hline 97. & Brassica rapa L. ssp. rapa & Brassicaceae & $\mathrm{T}$ & CUAD & & & CAS & $\operatorname{arc}$ & M & \\
\hline 98. & Briza maxima $\mathrm{L}$. & Poaceae & $\mathrm{T}$ & CIME & & & & & & \\
\hline 99. & $\begin{array}{l}\text { Bromus erectus Hudson ssp. } \\
\text { transilvanicus (Steud.) Asch. Et } \\
\text { Graebn }\end{array}$ & Poaceae & $\mathrm{H}$ & SEME & & & & & & \\
\hline 100. & Bromus hordeaceus L. ssp. hordeaceus & Poaceae & $\mathrm{T}$ & SEME & & & & & & \\
\hline
\end{tabular}




\begin{tabular}{|c|c|c|c|c|c|c|c|c|c|c|}
\hline 101. & Bromus madritensis L. & Poaceae & $\mathrm{T}$ & MEAT & & & & & & \\
\hline 102. & Bromus squarrosus L. & Poaceae & $\mathrm{T}$ & SEPO & & & & & & \\
\hline 103. & Bromus sterilis L. & Poaceae & $\mathrm{T}$ & WISP & & & & & & \\
\hline 104. & $\begin{array}{l}\text { Broussonetia papyrifera L'Herit ex } \\
\text { Vent. }\end{array}$ & Moraceae & $\mathrm{P}$ & CUAD & & & INV & neo & As-E & \\
\hline 105. & Bunias erucago L. & Brassicaceae & $\mathrm{T}$ & SEME & & & & & & \\
\hline 106. & $\begin{array}{l}\text { Bupleurum falcatum L. ssp. cernuum } \\
\text { (Ten.) Arcang. }\end{array}$ & Apiaceae & $\mathrm{H}$ & EURO & & & & & & BECK 1927 \\
\hline 107. & Bupleurum praealtum $\mathrm{L}$. & Apiaceae & $\mathrm{H}$ & EUAS & & & & & & BECK 1927 \\
\hline 108. & Bupleurum veronense Turra & Apiaceae & $\mathrm{T}$ & ILSE & & & & & & $\begin{array}{c}\text { STRUSC } \\
1880 \\
\end{array}$ \\
\hline 109. & Calamintha glandulosa (Req.) Benth. & Lamiaceae & $\mathrm{H}$ & SEPO & & & & & & BECK 1983 \\
\hline 110. & Calamintha sylvatica Bromf. & Lamiaceae & $\mathrm{H}$ & EURO & & & & & & BECK 1983 \\
\hline 111. & Calendula officinalis $\mathrm{L}$. & Asteraceae & $\mathrm{T}$ & CUAD & & & CAS & $\operatorname{arc}$ & \begin{tabular}{|l|} 
Un- \\
kno. \\
\end{tabular} & \\
\hline 112. & Calepina irregularis (Asso) Thell. & Brassicaceae & $\mathrm{T}$ & EURO & & & & & & \\
\hline 113. & Callitriche palustris $\mathrm{L}$. & Callitrichaceae & Hy & WISP & & & & & & \\
\hline 114. & Calystegia sepium (L.) R.Br. & Convolvulaceae & $\mathrm{H}$ & WISP & & & & & & \\
\hline 115. & Campanula bononiensis $\mathrm{L}$. & Campanulaceae & $\mathrm{H}$ & EUAS & & & & & & $\begin{array}{c}\text { STRUSC } \\
1880\end{array}$ \\
\hline 116. & Campanula erinus $\mathrm{L}$. & Campanulaceae & $\mathrm{T}$ & CIME & & & & & & \\
\hline 117. & Campanula lingulata Waldst. Et Kit. & Campanulaceae & $\mathrm{H}$ & SEME & & & & & & BECK 1983 \\
\hline 118. & Canabis sativa $\mathrm{L}$. & Cannabaceae & $\mathrm{T}$ & CUAD & & & NAT & $\operatorname{arc}$ & As-C & \\
\hline 119. & Capsella bursa - pastoris (L.) Med. & Brassicaceae & $\mathrm{H}$ & WISP & & & & & & \\
\hline 120. & Capsella rubella Reut & Brassicaceae & $\mathrm{T}$ & CIME & & & & & & \\
\hline 121. & Cardamine graeca $\mathrm{L}$. & Brassicaceae & $\mathrm{T}$ & EAME & & $\mathrm{CR}$ & & & & \\
\hline 122. & Cardamine hirsuta L. & Brassicaceae & $\mathrm{T}$ & WISP & & & & & & \\
\hline 123. & Cardamine maritima Port. ex DC. & Brassicaceae & $\mathrm{T}$ & ILAE & end & $\mathrm{CR}$ & & & & \\
\hline 124. & Cardaria draba (L.) Desv. & Brassicaceae & G & WISP & & & & & & \\
\hline 125. & Carduus micropterus (Borbás) Teyber & Asteraceae & $\mathrm{H}$ & ILAE & end & & & & & \\
\hline 126. & Carduus pycnocephalus L. & Asteraceae & $\mathrm{T}$ & CIME & & & & & & \\
\hline 127. & Carex caryophyllea Latourr. & Cyperaceae & $\mathrm{H}$ & EUAS & & & & & & \\
\hline 128. & Carex distachya Desf. & Cyperaceae & $\mathrm{H}$ & CIME & & & & & & \\
\hline 129. & Carex distans $\mathrm{L}$ & Cyperaceae & $\mathrm{H}$ & CIME & & & & & & \\
\hline 130. & Carex divulsa Stokes ssp. divulsa & Cyperaceae & $\mathrm{H}$ & WISP & & & & & & \\
\hline 131. & Carex flacca Schreber & Cyperaceae & G & WISP & & & & & & \\
\hline 132. & Carex hallerana Asso & Cyperaceae & $\mathrm{H}$ & SEME & & & & & & \\
\hline 133. & Carex hirta L. & Cyperaceae & G & EUAS & & & & & & \\
\hline 134. & Carex otrubae Podp. & Cyperaceae & $\mathrm{H}$ & SEME & & & & & & \\
\hline 135. & Carlina corymbosa $\mathrm{L}$. & Asteraceae & $\mathrm{T}$ & CIME & & & & & & $\begin{array}{c}\text { STRUSC } \\
1880\end{array}$ \\
\hline 136. & Carlina vulgaris L. ssp. vulgaris & Asteraceae & $\mathrm{T}$ & EUAS & & & & & & BECK 1983 \\
\hline
\end{tabular}




\begin{tabular}{|c|c|c|c|c|c|c|c|c|c|c|}
\hline 137. & Carpinus orientalis Mill & Corylaceae & $\mathrm{P}$ & ILSE & & & & & & \\
\hline 138. & Carthamus lanatus L. ssp. lanatus & Asteraceae & $\mathrm{T}$ & CIME & & & & & & \\
\hline 139. & Celtis australis $\mathrm{L}$. & Ulmaceae & $\mathrm{P}$ & SEME & & & & & & BECK 1916 \\
\hline 140. & Centaurea calcitrapa $\mathrm{L}$. & Asteraceae & $\mathrm{T}$ & MEAT & & & & & & \\
\hline 141. & $\begin{array}{l}\text { Centaurea deusta Ten. ssp. concolor } \\
\text { (DC.) Hayek }\end{array}$ & Asteraceae & $\mathrm{H}$ & EUME & & & & & & \\
\hline 142. & $\begin{array}{l}\text { Centaurea glaberrima Tausch ssp. } \\
\text { divergens (Vis.) Hayek }\end{array}$ & Asteraceae & $\mathrm{H}$ & ILAE & end & EN & & & & $\begin{array}{l}\text { STRUSCH- } \\
\text { KA } 1880\end{array}$ \\
\hline 143. & Centaurea jacea $\mathrm{L}$. & Asteraceae & $\mathrm{H}$ & EUAS & & & & & & \\
\hline 144. & $\begin{array}{l}\text { Centaurea rupestris L. ssp. ceratophylla } \\
\text { (Ten.) Gugler }\end{array}$ & Asteraceae & $\mathrm{H}$ & ILAE & end & $\mathrm{DD}$ & & & & \\
\hline 145. & Centaurea scabiosa $\mathrm{L}$. & Asteraceae & $\mathrm{H}$ & EUAS & & & & & & \\
\hline 146. & Centaurea solstitialis L. ssp.solstialis & Asteraceae & $\mathrm{T}$ & SEPO & & & & & & BECK 1983 \\
\hline 147. & Centaurium erythraea Rafn. & Gentianaceae & $\mathrm{T}$ & WISP & & & & & & \\
\hline 148. & $\begin{array}{l}\text { Cephalaria leucantha (L.) Roemer \& } \\
\text { Schultes }\end{array}$ & Dipsacaceae & $\mathrm{H}$ & CIME & & & & & & \\
\hline 149. & Cerastium glomeratum Thuill. & Caryophyllaceae & $\mathrm{T}$ & WISP & & & & & & \\
\hline 150. & $\begin{array}{l}\text { Cerastium ligusticum Viv. ssp. } \\
\text { trichogynum (Moschl) P.D.Sell. et } \\
\text { Whitehead }\end{array}$ & Caryophyllaceae & $\mathrm{T}$ & WEME & & & & & & \\
\hline 151. & Cercis siliquastrum $\mathrm{L}$. & Fabaceae & $\mathrm{P}$ & CUAD & & & CAS & arc & M & \\
\hline 152. & $\begin{array}{l}\text { Cerinthe minor L. ssp. auriculata } \\
\text { (Ten.) Domac }\end{array}$ & Boraginaceae & $\mathrm{H}$ & ILAP & end & NT & & & & BECK 1967 \\
\hline 153. & $\begin{array}{l}\text { Chaenorhinum minus (L.) Lange ssp. } \\
\text { minus }\end{array}$ & Scrophulariaceae & $\mathrm{T}$ & EURO & & & & & & \\
\hline 154. & Chaerophyllum coloratum $\mathrm{L}$. & Apiaceae & $\mathrm{H}$ & ILAE & end & EN & & & & \\
\hline 155. & Chamomilla recutita (L.) Rauschert & Asteraceae & $\mathrm{T}$ & WISP & & & & & & \\
\hline 156. & $\begin{array}{l}\text { Cheilanthes persica (Bory) Mett. ex } \\
\text { Kuhn }\end{array}$ & Adiantaceae & $\mathrm{H}$ & MEAT & & & & & & \\
\hline 157. & Chelidonium majus L. & Papaveraceae & $\mathrm{H}$ & WISP & & & & & & \\
\hline 158. & Chenopodium album $\mathrm{L}$. & Chenopodiaceae & $\mathrm{T}$ & WISP & & & & & & \\
\hline 159. & Chenopodium ambrosioides L. & Chenopodiaceae & $\mathrm{T}$ & CUAD & & & INV & neo & Am-T & \\
\hline 160. & Chenopodium botrys $\mathrm{L}$. & Chenopodiaceae & $\mathrm{T}$ & EUAS & & & & & & \\
\hline 161. & Chenopodium hybridum $\mathrm{L}$. & Chenopodiaceae & $\mathrm{T}$ & WISP & & & & & & \\
\hline 162. & Chondrilla juncea $\mathrm{L}$. & Asteraceae & $\mathrm{H}$ & EUAS & & & & & & $\begin{array}{l}\text { STRUSC } \\
1880\end{array}$ \\
\hline 163. & Chrozophora tinctoria (L.) Juss. & Euphorbiaceae & $\mathrm{T}$ & MEPO & & & & & & BECK 1920 \\
\hline 164. & Chrysopogon gryllus (L.) Trin & Poaceae & $\mathrm{H}$ & MEPO & & & & & & \\
\hline 165. & Cichorium intybus L. & Asteraceae & $\mathrm{H}$ & WISP & & & & & & \\
\hline 166. & Cirsium vulgare (Savi) Ten. & Asteraceae & $\mathrm{H}$ & EUAS & & & & & & \\
\hline 167. & Citrullus lanatus (Thunb.) Mansf & Cucurbitaceae & $\mathrm{T}$ & CUAD & & & CAS & $\operatorname{arc}$ & Af-S & \\
\hline 168. & Cleistogenes serotina (L.) Keng. & Poaceae & $\mathrm{H}$ & SEPO & & & & & & \\
\hline 169. & Clematis flammula $\mathrm{L}$. & Ranunculaceae & $\mathrm{P}$ & CIME & & & & & & BECK 1914 \\
\hline 170. & Clematis recta $\mathrm{L}$. & Ranunculaceae & $\mathrm{P}$ & EUAS & & & & & & \\
\hline 171. & Clematis vitalba $\mathrm{L}$. & Ranunculaceae & $\mathrm{P}$ & EURO & & & & & & \\
\hline
\end{tabular}




\begin{tabular}{|c|c|c|c|c|c|c|c|c|c|}
\hline 172. & Clematis viticella $\mathrm{L}$. & Ranunculaceae & $\mathrm{P}$ & SEME & & & & & BECK 1914 \\
\hline 173. & Clinopodium vulgare $\mathrm{L}$. & Lamiaceae & $\mathrm{H}$ & WISP & & & & & \\
\hline 174. & Clypeola jonthlaspi $\mathrm{L}$ & Brassicaceae & $\mathrm{T}$ & CIME & $\mathrm{DD}$ & & & & \\
\hline 175. & Colchicum autumnale $\mathrm{L}$. & Colchicaceae & G & CEEU & & & & & \\
\hline 176. & Colchicum hungaricum Janka & Colchicaceae & G & CIME & & & & & \\
\hline 177. & Colutea arborescens $\mathrm{L}$. & Fabaceae & $\mathrm{P}$ & CIME & & & & & BECK 1927 \\
\hline 178. & Consolida regalis S.F.Gray & Ranunculaceae & $\mathrm{T}$ & CUAD & & NAT & arc & M & \\
\hline 179. & Convolvulus arvensis $\mathrm{L}$. & Convolvulaceae & G & WISP & & & & & \\
\hline 180. & Convolvulus cantabrica $\mathrm{L}$. & Convolvulaceae & $\mathrm{Ch}$ & SEME & & & & & BECK 1927 \\
\hline 181. & Conyza bonariensis (L.) Cronq. & Asteraceae & $\mathrm{T}$ & CUAD & & INV & neo & Am-C & \\
\hline 182. & Conyza canadensis (L.) Cronq. & Asteraceae & $\mathrm{T}$ & CUAD & & INV & neo & Am-N & $\begin{array}{c}\text { STRUSC } \\
1880 \\
\end{array}$ \\
\hline 183. & Cornus mas L. & Cornaceae & $\mathrm{P}$ & SECO & & & & & \\
\hline 184. & Cornus sanguinea $\mathrm{L}$. & Cornaceae & $\mathrm{P}$ & EURO & & & & & \\
\hline 185. & $\begin{array}{l}\text { Coronilla emerus L. ssp. emeroides } \\
\text { (Boiss. \& Spruner) Hayek.. }\end{array}$ & Fabaceae & $\mathrm{P}$ & EAME & & & & & \\
\hline 186. & Coronilla scorpioides (L.) W.D.J.Koch. & Fabaceae & $\mathrm{T}$ & CIME & & & & & \\
\hline 187. & Coronilla varia $\mathrm{L}$. & Fabaceae & $\mathrm{H}$ & EURO & & & & & \\
\hline 188. & Cotinus coggygria Scop. & Anacardiaceae & $\mathrm{P}$ & SEPO & & & & & BECK 1921 \\
\hline 189. & Crataegus monogyna Jacqu. & Rosaceae & $\mathrm{P}$ & EUAS & & & & & \\
\hline 190. & Crepis foetida L. ssp. foetida & Asteraceae & $\mathrm{T}$ & SEME & & & & & \\
\hline 191. & Crepis neglecta $\mathrm{L}$ & Asteraceae & $\mathrm{T}$ & EUME & & & & & \\
\hline 192. & Crepis pannonica (Jacq.) C.Koch & Asteraceae & $\mathrm{T}$ & EEUP & $\mathrm{DD}$ & & & & $\begin{array}{c}\text { STRUSC } \\
1880\end{array}$ \\
\hline 193. & Crepis sancta (L.) Babc. & Asteraceae & $\mathrm{T}$ & EAME & & & & & \\
\hline 194. & Crepis vesicaria L. ssp. vesicaria & Asteraceae & $\mathrm{T}$ & CIME & & & & & \\
\hline 195. & Crocus reticulatus Steven ex Adams & Iridaceae & G & EEUP & & & & & \\
\hline 196. & Cruciata laevipes Opiz. & Rubiaceae & $\mathrm{H}$ & EUAS & & & & & \\
\hline 197. & $\begin{array}{l}\text { Cruciata pedemontana (Belliard) } \\
\text { Ehrend. }\end{array}$ & Rubiaceae & $\mathrm{H}$ & MEPO & & & & & $\begin{array}{l}\text { STRUSCH- } \\
\text { KA } 1880 \\
\end{array}$ \\
\hline 198. & Crupina vulgaris Cass. & Asteraceae & $\mathrm{T}$ & CIME & & & & & \\
\hline 199. & Cucurbita pepo L. & Cucurbitaceae & $\mathrm{T}$ & CUAD & & CAS & neo & Am-C & \\
\hline 200. & Cupressus sempervirens $\mathrm{L}$. & Cupressaceae & $\mathrm{P}$ & CUAD & & CAS & arc & M & \\
\hline 201. & Cuscuta campestris Yunker & Cuscutaceae & $\mathrm{T}$ & CUAD & & INV & neo & Am-N & \\
\hline 202. & Cuscuta epithymum L. & Cuscutaceae & $\mathrm{T}$ & WISP & & & & & \\
\hline 203. & Cyclamen hederifolium Aiton. & Primulaceae & G & SEME & $\mathrm{CR}$ & & & & \\
\hline 204. & $\begin{array}{l}\text { Cymbalaria muralis P.Gaertn., Mey. et } \\
\text { Scherb. }\end{array}$ & Scrophulariaceae & $\mathrm{H}$ & SEME & & & & & $\begin{array}{l}\text { STRUSCH- } \\
\text { KA } 1880 \\
\end{array}$ \\
\hline 205. & Cynodon dactylon (L.) Pers. & Poaceae & G & WISP & & & & & \\
\hline 206. & Cynoglossum columnae Ten. & Boraginaceae & $\mathrm{T}$ & EAME & $\mathrm{DD}$ & & & & \\
\hline 207. & Cynosurus echinatus L. & Poaceae & $\mathrm{T}$ & SEME & & & & & \\
\hline
\end{tabular}




\begin{tabular}{|c|c|c|c|c|c|c|c|c|c|c|}
\hline 208. & Cyperus longus $\mathrm{L}$. & Cyperaceae & Hy & WISP & & & & & & \\
\hline 209. & Dactylis glomerata L. ssp. glomerata & Poaceae & $\mathrm{H}$ & EUAS & & & & & & \\
\hline 210. & $\begin{array}{l}\text { Dactylis glomerata L. ssp. hispanica } \\
\text { (Roth.) Nyman }\end{array}$ & Poaceae & $\mathrm{H}$ & CIME & & & & & & \\
\hline 211. & Dasypyrum villosum (L.) P.Candargy & Poaceae & $\mathrm{T}$ & SEME & & & & & & BECK 1903 \\
\hline 212. & Datura stramonium $\mathrm{L}$. & Solanaceae & $\mathrm{T}$ & CUAD & & & INV & neo & Am-N & \\
\hline 213. & Daucus carota L. ssp. carota & Apiaceae & $\mathrm{H}$ & WISP & & & & & & \\
\hline 214 & Desmazeria rigida (L.) Tutin & Poaceae & $\mathrm{T}$ & MEAT & & & & & & BECK 1903 \\
\hline 215. & $\begin{array}{l}\text { Dianthus ciliatus Guss. ssp. } \\
\text { dalmaticus (Čelak) Hayek }\end{array}$ & Caryophyllaceae & $\mathrm{H}$ & ILAE & end & & & & & \\
\hline 216. & $\begin{array}{l}\text { Dianthus sylvestris Wulf. in Jacq. ssp. } \\
\text { longicaulis (Ten.) Greu. Et Burd. }\end{array}$ & Caryophyllaceae & $\mathrm{H}$ & WEME & & & & & & BECK 1916 \\
\hline 217. & $\begin{array}{l}\text { Dianthus sylvestris Wulfen in Jacq. } \\
\text { ssp. sylvestris }\end{array}$ & Caryophyllaceae & $\mathrm{H}$ & SEMO & & & & & & \\
\hline 218. & $\begin{array}{l}\text { Dianthus sylvestris Wulfen in Jacq. } \\
\text { ssp. tergestinus (Reichenb.) Hayek }\end{array}$ & Caryophyllaceae & $\mathrm{H}$ & ILAE & & VU & & & & \\
\hline 219. & Dichanthium ischaemum (L.) Roberty & Poaceae & $\mathrm{H}$ & SEME & & & & & & \\
\hline 220. & Dictamnus albus L. & Rutaceae & $\mathrm{Ch}$ & EUAS & & & & & & BECK 1916 \\
\hline 221. & Digitaria sanguinalis (L.) Scop. & Poaceae & $\mathrm{T}$ & WISP & & & & & & \\
\hline 222. & Diplotaxis tenuifolia (D.) DC. & Brassicaceae & $\mathrm{H}$ & WISP & & & & & & \\
\hline 223. & Dipsacus fullonum $\mathrm{L}$. & Dipsacaceae & $\mathrm{H}$ & CIME & & & & & & \\
\hline 224. & Dorycnium herbaceum Vill. & Fabaceae & $\mathrm{H}$ & SECO & & & & & & \\
\hline 225. & Ecballium elaterium (L.) Richard & Cucurbitaceae & $\mathrm{H}$ & CIME & & & & & & \\
\hline 226. & Echinochloa crus - galli (L) P. Beauv. & Poaceae & $\mathrm{T}$ & WISP & & & & & & \\
\hline 227. & Echium italicum L. & Boraginaceae & $\mathrm{T}$ & CIME & & & & & & \\
\hline 228. & Echium vulgare $\mathrm{L}$. & Boraginaceae & $\mathrm{H}$ & EURO & & & & & & BECK 1967 \\
\hline 229. & $\begin{array}{l}\text { Edraianthus tenuifolius (Waldst. \& } \\
\text { Kit.) A.DC. }\end{array}$ & Campanulaceae & $\mathrm{H}$ & ILAE & end & LC & & & & \\
\hline 230. & $\begin{array}{l}\text { Eleocharis palustris (L.) Roemer \& } \\
\text { Schultes }\end{array}$ & Cyperaceae & Hy & WISP & & & & & & \\
\hline 231. & Eleusine indica (L.) Gaertn. & Poaceae & $\mathrm{T}$ & CUAD & & & INV & neo & As & \\
\hline 232. & Elymus hispidus (Opiz.) Melderis & Poaceae & $\mathrm{H}$ & EUAS & & & & & & \\
\hline 233. & Elymus repens (L.) Gould & Poaceae & G & WISP & & & & & & \\
\hline 234. & $\begin{array}{l}\text { Ephedra fragilis Desf. ssp. } \\
\text { campylopoda (C. A. Mayer) Asch. et } \\
\text { Graeb. }\end{array}$ & Ephedraceae & $\mathrm{Ch}$ & EAME & & & & & & \\
\hline 235. & Ephedra major Host. ssp. major & Ephedraceae & $\mathrm{Ch}$ & EAME & & EN & & & & \\
\hline 236. & Epilobium dodonaei Vill. & Onagraceae & $\mathrm{Ch}$ & SEMO & & & & & & \\
\hline 237. & Epilobium hirsutum L. & Onagraceae & $\mathrm{H}$ & EUAS & & & & & & \\
\hline 238. & Epilobium parviflorum Schreb. & Onagraceae & $\mathrm{H}$ & EUAS & & & & & & \\
\hline 239. & Equisetum arvense $\mathrm{L}$. & Equisetaceae & G & $\mathrm{CIHO}$ & & & & & & \\
\hline 240. & Eragrostis cilianensis (All.) F.T.Hubb. & Poaceae & $\mathrm{T}$ & WISP & & & & & & \\
\hline 241. & Eragrostis minor Host. & Poaceae & $\mathrm{T}$ & CIME & & & & & & \\
\hline 242. & $\begin{array}{l}\text { Erigeron annuus (L.) Pers. ssp. } \\
\text { annuus }\end{array}$ & Asteraceae & $\mathrm{T}$ & CUAD & & & INV & neo & Am-N & \\
\hline
\end{tabular}




\begin{tabular}{|c|c|c|c|c|c|c|c|c|c|}
\hline 243. & Erodium acaule (L.) Becherer et Thell. & Geraniaceae & $\mathrm{H}$ & SEMO & & & & & \\
\hline 244. & Erodium cicutarium (L.) L.Her & Geraniaceae & $\mathrm{T}$ & WISP & & & & & \\
\hline 245. & Erophila verna (L.) Chevall. ssp. verna & Brassicaceae & $\mathrm{T}$ & CIME & & & & & $\begin{array}{l}\text { STRUSCH- } \\
\text { KA } 1880\end{array}$ \\
\hline 246. & $\begin{array}{l}\text { Eruca vesicaria (L.) Cav. ssp. sativa } \\
\text { (Mill.) Thell }\end{array}$ & Brassicaceae & $\mathrm{T}$ & CUAD & & CAS & $\operatorname{arc}$ & M & BECK 1916 \\
\hline 247. & Eryngium amethystinum $\mathrm{L}$. & Apiaceae & $\mathrm{H}$ & ILSE & & & & & \\
\hline 248. & Eryngium campestre $\mathrm{L}$. & Apiaceae & $\mathrm{H}$ & SEME & & & & & \\
\hline 249. & Erysimum linariifolium Tausch & Brassicaceae & $\mathrm{H}$ & ILSE & end & & & & \\
\hline 250. & Euonymus europaeus L & Celastraceae & $\mathrm{P}$ & EUAS & & & & & \\
\hline 251. & Euonymus verrucosa Scop. & Celastraceae & $\mathrm{P}$ & SEPO & & & & & \\
\hline 252. & Eupatorium cannabinum $\mathrm{L}$. & Asteraceae & $\mathrm{H}$ & EUAS & & & & & \\
\hline 253. & Euphorbia chamaesyce L. & Euphorbiaceae & $\mathrm{T}$ & SEME & & & & & \\
\hline 254. & $\begin{array}{l}\text { Euphorbia characias L. ssp wulfenii } \\
\text { (Hoppe ex Koch.) Radd-Sm. }\end{array}$ & Euphorbiaceae & $\mathrm{Ch}$ & ILAE & & & & & BECK 1920 \\
\hline 255. & Euphorbia cyparissias L. & Euphorbiaceae & $\mathrm{H}$ & EUAS & & & & & \\
\hline 256. & Euphorbia esula L. & & $\mathrm{H}$ & EUAS & & & & & BECK 1920 \\
\hline 257. & Euphorbia exigua L. & Euphorbiaceae & $\mathrm{T}$ & SEME & & & & & \\
\hline 258. & Euphorbia falcata $\mathrm{L}$. & Euphorbiaceae & $\mathrm{T}$ & SEME & & & & & BECK 1916 \\
\hline 259. & Euphorbia helioscopia L. & Euphorbiaceae & $\mathrm{T}$ & WISP & & & & & \\
\hline 260. & Euphorbia maculata L. & Euphorbiaceae & $\mathrm{T}$ & CUAD & & INV & neo & Am-N & \\
\hline 261. & Euphorbia platyphyllos L. & Euphorbiaceae & $\mathrm{T}$ & CIME & & & & & \\
\hline 262. & Euphorbia spinosa L. & Euphorbiaceae & $\mathrm{Ch}$ & CIME & & & & & BECK 1920 \\
\hline 263. & Fallopia baldschuanica (Regel) J.Holub & b Polygonaceae & $\mathrm{P}$ & CUAD & & CAS & neo & As-C & \\
\hline 264. & Fallopia convolvulus (L.) A. Löve & Polygonaceae & $\mathrm{T}$ & WISP & & & & & \\
\hline 265. & Ferulago campestris (Besser) Grecescu & Apiaceae & $\mathrm{H}$ & EEUP & & & & & \\
\hline 266. & $\begin{array}{l}\text { Festuca arundinacea Schreb. ssp. } \\
\text { arundinacea }\end{array}$ & Poaceae & $\mathrm{H}$ & EURO & & & & & \\
\hline 267. & Festuca pratensis Huds. & Poaceae & $\mathrm{H}$ & WISP & & & & & \\
\hline 268. & Ficus carica $\mathrm{L}$. & Moraceae & $\mathrm{P}$ & CIME & & & & & BECK 1906 \\
\hline 269. & Filago vulgaris Lam. & Asteraceae & $\mathrm{T}$ & SEPO & & & & & \\
\hline 270. & Filipendula vulgaris Moenck. & Rosaceae & $\mathrm{H}$ & EUAS & & & & & BECK 1927 \\
\hline 271. & Foeniculum vugare Miller & Apiaceae & G & CIME & & & & & $\begin{array}{c}\text { STRUSC } \\
1880\end{array}$ \\
\hline 272. & Fragaria vesca Ehrh. & Rosaceae & $\mathrm{H}$ & WISP & & & & & \\
\hline 273. & Frangula alnus Miller & Rhamnaceae & $\mathrm{P}$ & WISP & & & & & \\
\hline 274. & Frangula rupestris (Scop.) Schur. & Rhamnaceae & $\mathrm{P}$ & ILAE & & & & & \\
\hline 275. & Fraxinus angustifolia Vahl & Oleaceae & $\mathrm{P}$ & EEUP & & & & & \\
\hline 276. & Fraxinus ornus L. & Oleaceae & $\mathrm{P}$ & SEME & & & & & $\begin{array}{c}\text { STRUSC } \\
1880 \\
\end{array}$ \\
\hline 277. & $\begin{array}{l}\text { Fumana procumbens (Dunal) Gren. \& } \\
\text { Godr. }\end{array}$ & Cistaceae & $\mathrm{Ch}$ & SEME & & & & & \\
\hline 278. & Fumaria gaillardotii Boiss. & Fumariaceae & $\mathrm{T}$ & CIME & & & & & \\
\hline
\end{tabular}




\begin{tabular}{|c|c|c|c|c|c|c|c|c|c|c|}
\hline 279. & Fumaria officinalis L. & Fumariaceae & $\mathrm{T}$ & WISP & & & & & & \\
\hline 280. & Fumaria parviflora Lam. & Fumariaceae & $\mathrm{T}$ & WISP & & & & & & \\
\hline 281. & Gagea villosa (M.Bieb.) Sweet & Fumariaceae & G & EUAS & & & & & & \\
\hline 282. & Galanthus nivalis L. & Amaryllidaceae & G & EUAS & & LC & & & & \\
\hline 283. & Galeopsis angustifolia Hoffm. & Lamiaceae & $\mathrm{T}$ & EUAS & & & & & & \\
\hline 284. & Galinsoga parviflora Cav. & Asteraceae & $\mathrm{T}$ & CUAD & & & INV & neo & Am-S & \\
\hline 285. & Galium aparine $\mathrm{L}$. & Rubiaceae & $\mathrm{T}$ & WISP & & & & & & \\
\hline 286. & Galium corrudifolium Vill. & Rubiaceae & $\mathrm{H}$ & SEME & & & & & & \\
\hline 287. & Galium firmum Tausch & Rubiaceae & $\mathrm{Ch}$ & ILAE & end & & & & & \\
\hline 288. & Galium verum $\mathrm{L}$. & Rubiaceae & $\mathrm{H}$ & WISP & & & & & & \\
\hline 289. & Geranium columbinum $\mathrm{L}$. & Geraniaceae & $\mathrm{T}$ & EUAS & & & & & & \\
\hline 290. & Geranium dissectum $\mathrm{L}$. & Geraniaceae & $\mathrm{T}$ & WISP & & & & & & \\
\hline 291. & Geranium lucidum L. & Geraniaceae & $\mathrm{T}$ & MEAT & & & & & & \\
\hline 292. & Geranium molle L. ssp. molle & Geraniaceae & $\mathrm{T}$ & EAME & & & & & & \\
\hline 293. & $\begin{array}{l}\text { Geranium molle L. ssp. brutium } \\
\text { (Gasparr.) Graebn. }\end{array}$ & Geraniaceae & $\mathrm{T}$ & EAME & & & & & & \\
\hline 294. & Geranium purpureum Vill. & Geraniaceae & $\mathrm{T}$ & SEME & & & & & & \\
\hline 295. & Geranium pusillum $\mathrm{L}$. & Geraniaceae & $\mathrm{T}$ & EUAS & & & & & & \\
\hline 296. & Geranium pyrenaicum Burm. $\mathrm{f}$. & Geraniaceae & $\mathrm{H}$ & SEME & & & & & & \\
\hline 297. & Geranium robertianum $\mathrm{L}$. & Geraniaceae & $\mathrm{T}$ & WISP & & & & & & \\
\hline 298. & Geranium rotundifolium $\mathrm{L}$. & Geraniaceae & $\mathrm{T}$ & EUAS & & & & & & \\
\hline 299. & Geum urbanum $\mathrm{L}$. & Rosaceae & $\mathrm{H}$ & WISP & & & & & & \\
\hline 300. & Glechoma hederacea $\mathrm{L}$. & Lamiaceae & $\mathrm{Ch}$ & $\mathrm{CIHO}$ & & & & & & \\
\hline 301. & Glyceria plicata (Fr.) Fr. & Poaceae & Hy & WISP & & & & & & \\
\hline 302. & $\begin{array}{l}\text { Goniolimon dalmaticum (C.Presl.) } \\
\text { Richb. }\end{array}$ & Plumbaginaceae & $\mathrm{H}$ & ILAE & & DD & & & & BECK 1967 \\
\hline 303. & Gratiola officinalis L. & Scrophulariaceae & $\mathrm{H}$ & WISP & & & & & & \\
\hline 304. & Haplophyllum patavinum (L.) G.Don & Rutaceae & $\mathrm{Ch}$ & SEMO & & & & & & BECK 1920 \\
\hline 305. & Hedera helix L. & Araliaceae & $\mathrm{P}$ & EURO & & & & & & $\begin{array}{c}\text { STRUSC } \\
1880\end{array}$ \\
\hline 306. & Hedypnois cretica (L.) Dum.Cours. & Asteraceae & $\mathrm{T}$ & CIME & & & & & & $\begin{array}{l}\text { STRUSC } \\
1880\end{array}$ \\
\hline 307. & $\begin{array}{l}\text { Helianthemum nummularium (L.) } \\
\text { Mill. ssp. nummularium }\end{array}$ & Cistaceae & $\mathrm{Ch}$ & SEME & & & & & & \\
\hline 308. & Helianthus annuus L. & Asteraceae & $\mathrm{T}$ & CUAD & & & CAS & neo & Am-S & \\
\hline 309. & Helianthus tuberosus L. & Asteraceae & G & CUAD & & & INV & neo & Am-N & \\
\hline 310. & $\begin{array}{l}\text { Helichrysum italicum (Roth) Mill. } \\
\text { Corr. Guss. }\end{array}$ & Asteraceae & $\mathrm{Ch}$ & CIME & & & & & & \\
\hline 311. & Heliotropium europaeum $\mathrm{L}$. & Boraginaceae & $\mathrm{T}$ & MEPO & & & & & & $\begin{array}{c}\text { STRUSC } \\
1880\end{array}$ \\
\hline 312. & Heliotropium supinum $\mathrm{L}$. & Boraginaceae & $\mathrm{T}$ & ILSE & & & & & & BECK 1967 \\
\hline 313. & Hermodactylus tuberosus (L.) Mill & Iridaceae & G & EAME & & CR & & & & \\
\hline 314. & Herniaria hirsuta L. & Caryopyllaceae & $\mathrm{T}$ & EURO & & & & & & \\
\hline
\end{tabular}




\begin{tabular}{|c|c|c|c|c|c|c|c|c|c|c|}
\hline 315. & Herniaria incana Lam. & Caryopyllaceae & $\mathrm{Ch}$ & SEME & & & & & & BECK 1906 \\
\hline 316. & Hesperis laciniata All. & Brassicaceae & $\mathrm{H}$ & ILSE & & & & & & \\
\hline 317. & $\begin{array}{l}\text { Hordeum murinum L. ssp. leporinum } \\
\text { (Link) Arcang }\end{array}$ & Poaceae & $\mathrm{T}$ & CIME & & & & & & \\
\hline 318. & Hordeum vulgare $\mathrm{L}$. & Poaceae & $\mathrm{T}$ & CUAD & & & CAS & arc & $\begin{array}{l}\text { Un- } \\
\text { kno. }\end{array}$ & \\
\hline 319. & Hornungia petraea (L.) Rchb. & Brassicaceae & $\mathrm{T}$ & EUME & & & & & & \\
\hline 320. & Humulus lupulus L. & Cannabaceae & $\mathrm{P}$ & EUAS & & & & & & \\
\hline 321. & Hyacinthus orientalis $\mathrm{L}$. & Liliaceae & & CUAD & & & CAS & arc & M & \\
\hline 322. & Hyoscyamus niger $\mathrm{L}$. & Solanaceae & $\mathrm{T}$ & EUAS & & & & & & \\
\hline 323. & Hyoscyamus albus L. & Solanaceae & $\mathrm{T}$ & CIME & & DD & & & & $\begin{array}{c}\text { STRUSC } \\
1880\end{array}$ \\
\hline 324. & Hypericum perforatum $\mathrm{L}$. & Clusiaceae & $\mathrm{H}$ & WISP & & & & & & MALY 1905 \\
\hline 325. & Hypericum tetrapterum $\mathrm{Fr}$. & Clusiaceae & $\mathrm{H}$ & WISP & & & & & & \\
\hline 326. & Iberis umbellata $\mathrm{L}$. & Brassicaceae & $\mathrm{T}$ & ILAE & & & & & & \\
\hline 327. & Inula britannica $\mathrm{L}$. & Asteraceae & $\mathrm{H}$ & EURO & & & & & & \\
\hline 328. & Inula conyza DC. & Asteraceae & $\mathrm{H}$ & SEPO & & & & & & \\
\hline 329. & Inula ensifolia $\mathrm{L}$. & Asteraceae & $\mathrm{H}$ & SEPO & & & & & & \\
\hline 330. & Inula spiraeifolia $\mathrm{L}$. & Asteraceae & $\mathrm{H}$ & SEME & & & & & & \\
\hline 331. & Inula verbascifolia (Willd.) Hausskn. & Asteraceae & $\mathrm{H}$ & ILAE & & & & & & MALY 1905 \\
\hline 332. & Ipomaea purpurea Roth. & Convolvulaceae & $\mathrm{T}$ & CUAD & & & CAS & neo & A-S & \\
\hline 333. & Iris germanica $\mathrm{L}$. & Iridaceae & $\mathrm{H}$ & CUAD & & & NAT & arc & As-E & \\
\hline 334. & Iris pseudopallida Trinajstić & Iridaceae & G & ILAE & end & DD & & & & \\
\hline 335. & Juncus articulatus L. & Juncaceae & G & $\mathrm{CIHO}$ & & & & & & \\
\hline 336. & Juncus bufonius L. & Juncaceae & $\mathrm{T}$ & WISP & & & & & & \\
\hline 337. & Juncus inflexus L. & Juncaceae & $\mathrm{H}$ & EUAS & & & & & & \\
\hline 338. & Juniperus oxycedrus L. ssp. oxycedrus & Cupressaceae & $\mathrm{P}$ & CIME & & & & & & BECK 1903 \\
\hline 339. & Kickxia spuria (L.) Dum. & Scrophulariaceae & $\mathrm{T}$ & CUAD & & & NAT & arc & M & \\
\hline 340. & Knautia integrifolia (L.) Bertol. & Dipsacaceae & $\mathrm{T}$ & CIME & & & & & & \\
\hline 341. & Koeleria splendens C.Presl & Poaceae & $\mathrm{H}$ & SEME & & & & & & \\
\hline 342. & Lactuca saligna L. & Asteraceae & $\mathrm{T}$ & EURO & & & & & & \\
\hline 343. & Lactuca sativa $\mathrm{L}$. & Asteraceae & $\mathrm{H}$ & CUAD & & & CAS & arc & $\begin{array}{l}\text { Un- } \\
\text { kno. }\end{array}$ & \\
\hline 344. & Lactuca serriola $\mathrm{L}$. & Asteraceae & $\mathrm{T}$ & WISP & & & & & & \\
\hline 345. & Lactuca viminea ( L.) J.Presl \& C.Presl & Asteraceae & $\mathrm{H}$ & SEPO & & & & & & \\
\hline 346. & Lamium amplexicaule $\mathrm{L}$. & Lamiaceae & $\mathrm{T}$ & EUAS & & & & & & \\
\hline 347. & Lamium maculatum $\mathrm{L}$. & Lamiaceae & $\mathrm{H}$ & EUAS & & & & & & BECK 1974 \\
\hline 348. & Lamium purpureum L. & Lamiaceae & $\mathrm{T}$ & EUAS & & & & & & \\
\hline 349. & $\begin{array}{l}\text { Lappula squarrosa (Retz.) Dumort. } \\
\text { subsp. squarrosa }\end{array}$ & Boraginaceae & $\mathrm{T}$ & EURO & & & & & & \\
\hline 350. & Lapsana communis L. & Asteraceae & $\mathrm{T}$ & WISP & & & & & & \\
\hline
\end{tabular}




\begin{tabular}{|c|c|c|c|c|c|c|c|c|c|c|}
\hline 351. & Lathyrus aphaca L & Fabaceae & $\mathrm{T}$ & SEME & & & & & & \\
\hline 352. & Lathyrus cicera $\mathrm{L}$. & Fabaceae & $\mathrm{T}$ & CIME & & & & & & \\
\hline 353. & Lathyrus latifolius L. & Fabaceae & $\mathrm{H}$ & SEME & & & & & & \\
\hline 354. & Lathyrus setifolius L. & Fabaceae & $\mathrm{T}$ & MEPO & & & & & & \\
\hline 355. & Lathyrus sphaericus Retz. & Fabaceae & $\mathrm{T}$ & CIME & & & & & & \\
\hline 356. & Lathyrus tuberosus L. & Fabaceae & $\mathrm{H}$ & EUAS & & & & & & \\
\hline 357. & Laurus nobilis L. & Lauraceae & $\mathrm{P}$ & CUAD & & & CAS & $\operatorname{arc}$ & M & \\
\hline 358. & Lavatera cretica $\mathrm{L}$. & Malvaceae & $\mathrm{H}$ & CIME & & & & & & \\
\hline 359. & Lavatera thuringiaca $\mathrm{L}$. & Malvaceae & $\mathrm{H}$ & EUAS & & & & & & \\
\hline 360. & Legousia hybrida (L.) Delarbre & Campanulaceae & $\mathrm{T}$ & SEAT & & NT & & & & \\
\hline 361. & $\begin{array}{l}\text { Legousia speculum veneris (L.) Chaix } \\
\text { in Vill. }\end{array}$ & Campanulaceae & $\mathrm{T}$ & SEME & & & & & & \\
\hline 362. & $\begin{array}{l}\text { Leontodon hispidus L. ssp. danubialis } \\
\text { (Jacq.) Simonk. }\end{array}$ & Asteraceae & $\mathrm{H}$ & SEPO & & & & & & $\begin{array}{l}\text { STRUSCH- } \\
\text { KA } 1880\end{array}$ \\
\hline 363. & Leontodon tuberosus L. & Asteraceae & $\mathrm{H}$ & CIME & & & & & & \\
\hline 364. & Lepidium graminifolium $\mathrm{L}$. & Brassicaceae & $\mathrm{H}$ & SEPO & & & & & & \\
\hline 365. & Lepidium ruderale $\mathrm{L}$. & Brassicaceae & $\mathrm{T}$ & EUAS & & & & & & \\
\hline 366. & Ligustrum vulgare $\mathrm{L}$. & Oleaceae & $\mathrm{P}$ & CEEU & & & & & & $\begin{array}{l}\text { STRUSC } \\
1880\end{array}$ \\
\hline 367. & $\begin{array}{l}\text { Linaria genistifolia (L.) Mill. subsp. } \\
\text { dalmatica (L.) Maire \& Petitm. }\end{array}$ & Scrophulariaceae & $\mathrm{H}$ & BAAP & & & & & & BECK 1967 \\
\hline 368. & Linaria microsepala Kerner. & Scrophulariaceae & $\mathrm{T}$ & ILAE & end & DD & & & & MALY 1905 \\
\hline 369. & Linaria vulgaris Miller. & Scrophulariaceae & $\mathrm{H}$ & EUAS & & & & & & \\
\hline 370. & Linum tenuifolium $\mathrm{L}$. & Linaceae & $\mathrm{H}$ & SEPO & & & & & & $\begin{array}{l}\text { STRUSC } \\
1880\end{array}$ \\
\hline 371. & Lithospermum arvense $\mathrm{L}$. & Boraginaceae & $\mathrm{Ch}$ & EUAS & & & & & & \\
\hline 372. & Lithospermum purpurocaeruleum $\mathrm{L}$. & Boraginaceae & $\mathrm{Ch}$ & SEPO & & & & & & \\
\hline 373. & Lolium multiflorum Lam. & Poaceae & $\mathrm{T}$ & CIME & & & & & & \\
\hline 374. & Lolium perenne $\mathrm{L}$ & Poaceae & $\mathrm{H}$ & EURO & & & & & & \\
\hline 375. & Lonicera etrusca Santi & Caprifoliaceae & $\mathrm{P}$ & CIME & & & & & & \\
\hline 376. & Lophochloa cristata (L.) Hyl. & Poaceae & $\mathrm{H}$ & MEAT & & & & & & \\
\hline 377. & $\begin{array}{l}\text { Lotus corniculatus L. ssp. hirsutus } \\
\text { Rothm. }\end{array}$ & Fabaceae & $\mathrm{H}$ & SEME & & & & & & \\
\hline 378. & Lunaria annua $\mathrm{L}$. & Brassicaceae & $\mathrm{H}$ & SEEU & & & & & & \\
\hline 379. & Lycopus europaeus L. & Lamiaceae & $\mathrm{H}$ & EUAS & & & & & & \\
\hline 380. & Lysimachia nummularia $\mathrm{L}$. & Primulaceae & $\mathrm{H}$ & EURO & & & & & & $\begin{array}{c}\text { STRUSC } \\
1880\end{array}$ \\
\hline 381. & Lysimachia vulgaris $\mathrm{L}$. & Primulaceae & $\mathrm{H}$ & EUAS & & & & & & BECK 1967 \\
\hline 382. & Lythrum salicaria $\mathrm{L}$. & Lythraceae & $\mathrm{H}$ & WISP & & & & & & \\
\hline 383. & $\begin{array}{l}\text { Maclura pomifera (Rafin.) C.K. } \\
\text { Schneider }\end{array}$ & Moraceae & $\mathrm{P}$ & CUAD & & & CAS & neo & Am-N & \\
\hline 384. & Malva neglecta Wallr. & Malvaceae & $\mathrm{T}$ & WISP & & & & & & \\
\hline 385. & Malva sylvestris L. & Malvaceae & $\mathrm{H}$ & WISP & & & & & & \\
\hline
\end{tabular}




\begin{tabular}{|c|c|c|c|c|c|c|c|c|c|c|}
\hline 386. & Marrubium incanum Desr. & Lamiaceae & $\mathrm{H}$ & ILAP & & & & & & BECK 1950 \\
\hline 387. & Marrubium vulgare $\mathrm{L}$. & Lamiaceae & $\mathrm{H}$ & WISP & & & & & & BECK 1950 \\
\hline 388. & Medicago arabica (L.) Huds. & Fabaceae & $\mathrm{T}$ & CUAD & & & NAT & arc & M & \\
\hline 389. & Medicago falcata $\mathrm{L}$. & Fabaceae & $\mathrm{H}$ & EUAS & & & & & & \\
\hline 390. & Medicago lupulina L. & Fabaceae & $\mathrm{T}$ & WISP & & & & & & \\
\hline 391. & Medicago minima (L.) Bartal & Fabaceae & $\mathrm{T}$ & WISP & & & & & & \\
\hline 392. & Medicago orbicularis (L.) Bartal & Fabaceae & $\mathrm{T}$ & CIME & & & & & & \\
\hline 393. & Medicago polymorpha L. & Fabaceae & $\mathrm{T}$ & SEME & & & & & & \\
\hline 394. & Medicago prostrata Jacq. & Fabaceae & $\mathrm{H}$ & SEME & & & & & & \\
\hline 395. & Medicago rigidula (L.) All & Fabaceae & $\mathrm{H}$ & MEPO & & & & & & \\
\hline 396. & Medicago sativa $\mathrm{L}$. & Fabaceae & $\mathrm{H}$ & CUAD & & & NAT & arc & Unkno & \\
\hline 397. & Melampyrum fimbriatum Vand. & Scrophulariaceae & $\mathrm{T}$ & ILAP & end & $\mathrm{DD}$ & & & & MALY 1920 \\
\hline 398. & Melia azedarach $\mathrm{L}$. & Meliaceae & $\mathrm{P}$ & CUAD & & & CAS & neo & As-C & \\
\hline 399. & Melica ciliata L. ssp. ciliata & Poaceae & $\mathrm{H}$ & EUAS & & & & & & \\
\hline 400. & Melilotus albus Medik & Fabaceae & $\mathrm{T}$ & EUAS & & & & & & \\
\hline 401. & Melilotus indica (L.) All. & Fabaceae & $\mathrm{T}$ & CIME & & & & & & \\
\hline 402. & Melissa officinalis L. & Lamiaceae & $\mathrm{H}$ & EAME & & & & & & \\
\hline 403. & Mentha aquatica L. & Lamiaceae & $\mathrm{H}$ & WISP & & & & & & \\
\hline 404. & Mentha longifolia (L.) Huds. & Lamiaceae & $\mathrm{H}$ & WISP & & & & & & \\
\hline 405. & Mentha pulegium $\mathrm{L}$. & Lamiaceae & $\mathrm{H}$ & EUME & & & & & & \\
\hline 406. & Mentha spicata $\mathrm{L}$. & Lamiaceae & $\mathrm{H}$ & WISP & & & & & & \\
\hline 407. & Mercurialis annua L. & Euphorbiaceae & $\mathrm{T}$ & WISP & & & & & & \\
\hline 408. & $\begin{array}{l}\text { Micromeria juliana (L.) Benth. ex } \\
\text { Rchb. }\end{array}$ & Lamiaceae & $\mathrm{Ch}$ & CIME & & & & & & BECK 1983 \\
\hline 409. & Micromeria thymifolia (Scop.) Fritsch. & Lamiaceae & $\mathrm{Ch}$ & ILAE & end & & & & & MALY 1923 \\
\hline 410. & Mirabilis jalapa $\mathrm{L}$. & Nyctaginaceae & G & CUAD & & & CAS & neo & Am-T & \\
\hline 411. & Misopates orontium (L.) Raf. & Scrophulariaceae & $\mathrm{T}$ & EUAS & & & & & & $\begin{array}{c}\text { STRUSC } \\
1880\end{array}$ \\
\hline 412. & $\begin{array}{l}\text { Molinia caerulea (L.) subsp. } \\
\text { arundinacea (Sch.) H.K.G. Paul }\end{array}$ & Poaceae & $\mathrm{H}$ & EUAS & & & & & & \\
\hline 413. & Moltkia petraea (Tratt.) Griseb. & Boraginaceae & $\mathrm{Ch}$ & ILAE & end & NT & & & & \\
\hline 414. & Morus alba L. & Moraceae & $\mathrm{P}$ & CUAD & & & NAT & arc & As-E & \\
\hline 415. & Morus nigra L. & Moraceae & $\mathrm{P}$ & CUAD & & & CAS & $\operatorname{arc}$ & As-SW & \\
\hline 416. & Muscari comosum (L.) Mill. & Liliaceae & G & SEME & & & & & & BECK 1903 \\
\hline 417. & Muscari neglectum Guss. ex Ten. & Liliaceae & G & CIME & & & & & & \\
\hline 418. & Mycelis muralis L. & Asteraceae & $\mathrm{H}$ & EUAS & & & & & & \\
\hline 419. & Myosotis arvensis (L.) Hill & Boraginaceae & $\mathrm{T}$ & EUAS & & & & & & BECK 1967 \\
\hline 420. & Myosotis ramosissima Rochel & Boraginaceae & $\mathrm{T}$ & EUAS & & & & & & \\
\hline 421. & Myosotis scorpioides L. & Boraginaceae & $\mathrm{H}$ & $\mathrm{CIHO}$ & & & & & & \\
\hline 422. & Myosotis sylvatica Hoffm. & Boraginaceae & $\mathrm{H}$ & EUAS & & & & & & BECK 1967 \\
\hline
\end{tabular}




\begin{tabular}{|c|c|c|c|c|c|c|c|c|c|c|}
\hline 423. & Myriophyllum verticillatum $\mathrm{L}$. & Haloragaceae & $\mathrm{Hy}$ & $\mathrm{CIHO}$ & & & & & & \\
\hline 424 & Nasturtium officinale $\mathrm{R}$. Br & Brassicaceae & $\mathrm{H}$ & WISP & & & & & & \\
\hline 425. & Nepeta cataria L. & Lamiaceae & $\mathrm{Ch}$ & EAME & & & & & & BECK 1950 \\
\hline 426. & Nigella damascena L. & Ranunculaceae & $\mathrm{T}$ & CIME & & & & & & BECK 1907 \\
\hline 427. & Oenanthe fistulosa $\mathrm{L}$. & Apiaceae & $\mathrm{H}$ & WISP & & & & & & \\
\hline 428. & Oenanthe pimpinelloides $\mathrm{L}$. & Apiaceae & $\mathrm{H}$ & MEAT & & & & & & \\
\hline 429. & Oenanthera biennis L. & Onagraceae & $\mathrm{H}$ & CUAD & & & INV & neo & Am-N & \\
\hline 430. & $\begin{array}{l}\text { Onobrychis arenaria (Kit.) DC. ssp. } \\
\text { tommasinii (Jord.) Asch. Et Graebn. }\end{array}$ & Fabaceae & $\mathrm{H}$ & EUAS & & & & & & \\
\hline 431. & Onobrychis caput-galli (L.) Lam. & Fabaceae & $\mathrm{T}$ & CIME & & & & & & \\
\hline 432. & Ononis antiquorum (L.) Arcang. & Fabaceae & $\mathrm{Ch}$ & CIME & & & & & & \\
\hline 433. & Onopordum Illyricum L. & Asteraceae & $\mathrm{H}$ & CIME & & & & & & \\
\hline 434 & Onosma echioides L. & Boraginaceae & $\mathrm{Ch}$ & ILAE & end & & & & & \\
\hline 435. & Ophrys bertolonii Moretti & Orchidaceae & G & BAAP & & & & & & \\
\hline 436. & $\begin{array}{l}\text { Ophrys sphegodes Mill. subsp. atrata } \\
\text { (Lind1.) E.Mayer }\end{array}$ & Orchidaceae & G & EUME & & & & & & \\
\hline 437. & Opopanax chironium (L.) W.D.J.Koch & Apiaceae & $\mathrm{H}$ & CIME & & EN & & & & ŠILIĆ, 1972 \\
\hline 438. & Orchis morio L. ssp. morio & Orchidaceae & G & EUME & & & & & & \\
\hline 439. & Origanum vulgare $\mathrm{L}$. & Lamiaceae & $\mathrm{H}$ & EUAS & & & & & & $\begin{array}{c}\text { STRUSC } \\
1880\end{array}$ \\
\hline 440 & Orlaya grandiflora (L.) Hoffm. & Apiaceae & $\mathrm{T}$ & SEME & & & & & & \\
\hline 441. & Ornithogalum gussonei Ten. & Liliaceae & G & SEEU & & & & & & \\
\hline 442. & Ornithogalum sphaerocarpum A.Kern. & Liliaceae & G & SEME & & & & & & \\
\hline 443. & Orobanche caryophyllacea Sm. & Orobanchaceae & $\mathrm{T}$ & SEME & & & & & & \\
\hline 444. & Orobanche hederae Duby. & Orobanchaceae & $\mathrm{T}$ & SEME & & & & & & \\
\hline 445. & Orobanche minor $\mathrm{Sm}$. & Orobanchaceae & $\mathrm{T}$ & SEME & & & & & & \\
\hline 446. & Orobanche reticulata Wallr. & Orobanchaceae & $\mathrm{T}$ & EURO & & & & & & \\
\hline 447. & Ostrya carpinifolia Scop & Corylaceae & $\mathrm{P}$ & ILSE & & & & & & \\
\hline 448. & Osyris alba L. & Santalaceae & $P$ & CIME & & & & & & \\
\hline 449. & Oxalis articulata Savigny & Oxalidaceae & G & CUAD & & & CAS & neo & Am-S & \\
\hline 450. & Oxalis corniculata $\mathrm{L}$. & Oxalidaceae & $\mathrm{T}$ & WISP & & & & & & $\begin{array}{c}\text { STRUSC } \\
1880\end{array}$ \\
\hline 451. & Oxalis dillenii Jacq. & Oxalidaceae & $\mathrm{H}$ & CUAD & & & NAT & neo & Am-N & \\
\hline 452. & Paliurus spina - christi Mill. & Rhamnaceae & $\mathrm{T}$ & ILSE & & & & & & BECK 1921 \\
\hline 453. & Panicum miliaceum $\mathrm{L}$. & Poaceae & $\mathrm{T}$ & CUAD & & & NAT & arc & As-C & \\
\hline 454. & Papaver rhoeas L. & Papaveraceae & $\mathrm{T}$ & CUAD & & & NAT & $\operatorname{arc}$ & M & \\
\hline 455. & Parentucellia latifolia (L.) Caruel & Scrophulariaceae & $\mathrm{T}$ & CIME & & & & & & BECK 1967 \\
\hline 456. & Parietaria judaica L. & Urticaceae & $\mathrm{H}$ & SEME & & & & & & \\
\hline 457. & Parietaria officinalis L. & Urticaceae & $\mathrm{H}$ & EUAS & & & & & & \\
\hline 458. & Paronychia kapela (Hacq.) a.Kern. & Caryophyllaceae & $\mathrm{H}$ & SEME & & & & & & \\
\hline
\end{tabular}




\begin{tabular}{|c|c|c|c|c|c|c|c|c|c|c|}
\hline 459. & $\begin{array}{l}\text { Parthenocissus quinquefolia (L.) } \\
\text { Planchon. }\end{array}$ & Vitaceae & $\mathrm{P}$ & CUAD & & & INV & neo & Am-N & \\
\hline 460. & Pastinacia sativa $\mathrm{L}$. & Apiaceae & $\mathrm{H}$ & EUAS & & & & & & \\
\hline 461. & $\begin{array}{l}\text { Petrorhagia prolifera (L.) P.w. Ball \& } \\
\text { Heywood }\end{array}$ & Caryophyllaceae & $\mathrm{T}$ & CIME & & & & & & \\
\hline 462. & Petrorhagia saxifraga (L.) Link & Caryophyllaceae & $\mathrm{H}$ & SEME & & & & & & \\
\hline 463. & Petteria ramentacea (Sieb) Presl. & Fabaceae & $\mathrm{P}$ & ILAE & end & NT & & & & $\begin{array}{c}\text { STRUSC } \\
1880\end{array}$ \\
\hline 464. & Peucedanum oreoselinum (L.) Moench & Apiaceae & $\mathrm{H}$ & SEME & & & & & & \\
\hline 465. & Phalaris arundinacea $\mathrm{L}$. & Poaceae & $\mathrm{H}$ & $\mathrm{CIHO}$ & & & & & & \\
\hline 466. & Phalaris canariensis $\mathrm{L}$. & Poaceae & $\mathrm{T}$ & CUAD & & & NAT & neo & Af & \\
\hline 467. & Phillyrea latifolia L. & Oleaceae & $\mathrm{P}$ & CIME & & & & & & $\begin{array}{c}\text { STRUSC } \\
1880 \\
\end{array}$ \\
\hline 468. & Phleum echinatum Host & Poaceae & $\mathrm{T}$ & CIME & & & & & & \\
\hline 469. & $\begin{array}{l}\text { Phleum pratense L. ssp. bertolonii } \\
\text { (DC.) Borum. }\end{array}$ & Poaceae & $\mathrm{H}$ & EUAS & & & & & & \\
\hline 470. & Phleum pratense L. ssp. pratense & Poaceae & $\mathrm{H}$ & $\mathrm{CIHO}$ & & & & & & \\
\hline 471. & $\begin{array}{l}\text { Phleum subulatum (Savi) Asch. \& } \\
\text { Graebn. }\end{array}$ & Poaceae & $\mathrm{T}$ & CIME & & & & & & \\
\hline 472. & $\begin{array}{l}\text { Phragmites australis (Cav.) Trin. ex } \\
\text { Steud. }\end{array}$ & Poaceae & Hy & WISP & & & & & & \\
\hline 473. & Physalis alkekengi $\mathrm{L}$. & Solanaceae & $\mathrm{H}$ & EURO & & & & & & \\
\hline 474. & Phytolacca americana L. & Phytolaccaceae & G & CUAD & & & INV & neo & Am-N & \\
\hline 475. & Picnomon acarna (L.) Cass. & Asteraceae & $\mathrm{T}$ & CIME & & & & & & \\
\hline 476. & Picris echioides L. & Asteraceae & $\mathrm{T}$ & CIME & & & & & & \\
\hline 477. & Picris hieracioides L. & Asteraceae & $\mathrm{H}$ & EUAS & & & & & & \\
\hline 478. & Pimpinella peregrina $\mathrm{L}$. & Apiaceae & $\mathrm{H}$ & SEME & & & & & & \\
\hline 479. & Pimpinella saxifraga $\mathrm{L}$. & Apiaceae & $\mathrm{H}$ & EUAS & & & & & & \\
\hline 480. & $\begin{array}{l}\text { Piptatherum holciforme (M.Bieb.) } \\
\text { Roem. \& Schult. }\end{array}$ & Poaceae & $\mathrm{H}$ & SEEU & & & & & & \\
\hline 481. & $\begin{array}{l}\text { Piptatherum miliaceum (L.) Coss. ssp. } \\
\text { thomasii (Duby) Soják }\end{array}$ & Poaceae & $\mathrm{H}$ & CIME & & & & & & \\
\hline 482. & Pistacia terebinthus L. & Anacardiaceae & $\mathrm{P}$ & CIME & & & & & & BECK 1921 \\
\hline 483. & Pisum sativum $\mathrm{L}$. & Fabaceae & $\mathrm{T}$ & CUAD & & & CAS & $\operatorname{arc}$ & $\begin{array}{l}\text { Un- } \\
\text { kno. }\end{array}$ & \\
\hline 484. & Plantago argentea Chaix. & Plantaginaceae & $\mathrm{H}$ & SEME & & & & & & $\begin{array}{c}\text { STRUSC } \\
1880 \\
\end{array}$ \\
\hline 485. & Plantago holosteum Scop. & Plantaginaceae & $\mathrm{H}$ & CIME & & & & & & \\
\hline 486. & Plantago lanceolata $\mathrm{L}$. & Plantaginaceae & $\mathrm{H}$ & WISP & & & & & & \\
\hline 487. & Plantago major L. ssp. major & Plantaginaceae & $\mathrm{H}$ & WISP & & & & & & \\
\hline 488. & Plantago media $\mathrm{L}$. & Plantaginaceae & $\mathrm{H}$ & EUAS & & & & & & \\
\hline 489. & Platanus acerifolia (Aiton) Willd. & Platanaceae & $\mathrm{P}$ & CUAD & & & NAT & neo & $\begin{array}{l}\text { Un- } \\
\text { kno. }\end{array}$ & \\
\hline 490. & Platanus orientalis $\mathrm{L}$. & Platanaceae & $\mathrm{P}$ & CUAD & & & CAS & $\operatorname{arc}$ & As & $\begin{array}{c}\text { STRUSC } \\
1880\end{array}$ \\
\hline 491. & Plumbago europaea $\mathrm{L}$. & Plumbaginaceae & $\mathrm{Ch}$ & CIME & & & & & & \\
\hline 492. & Poа аппиа L. & Poaceae & $\mathrm{T}$ & WISP & & & & & & \\
\hline
\end{tabular}




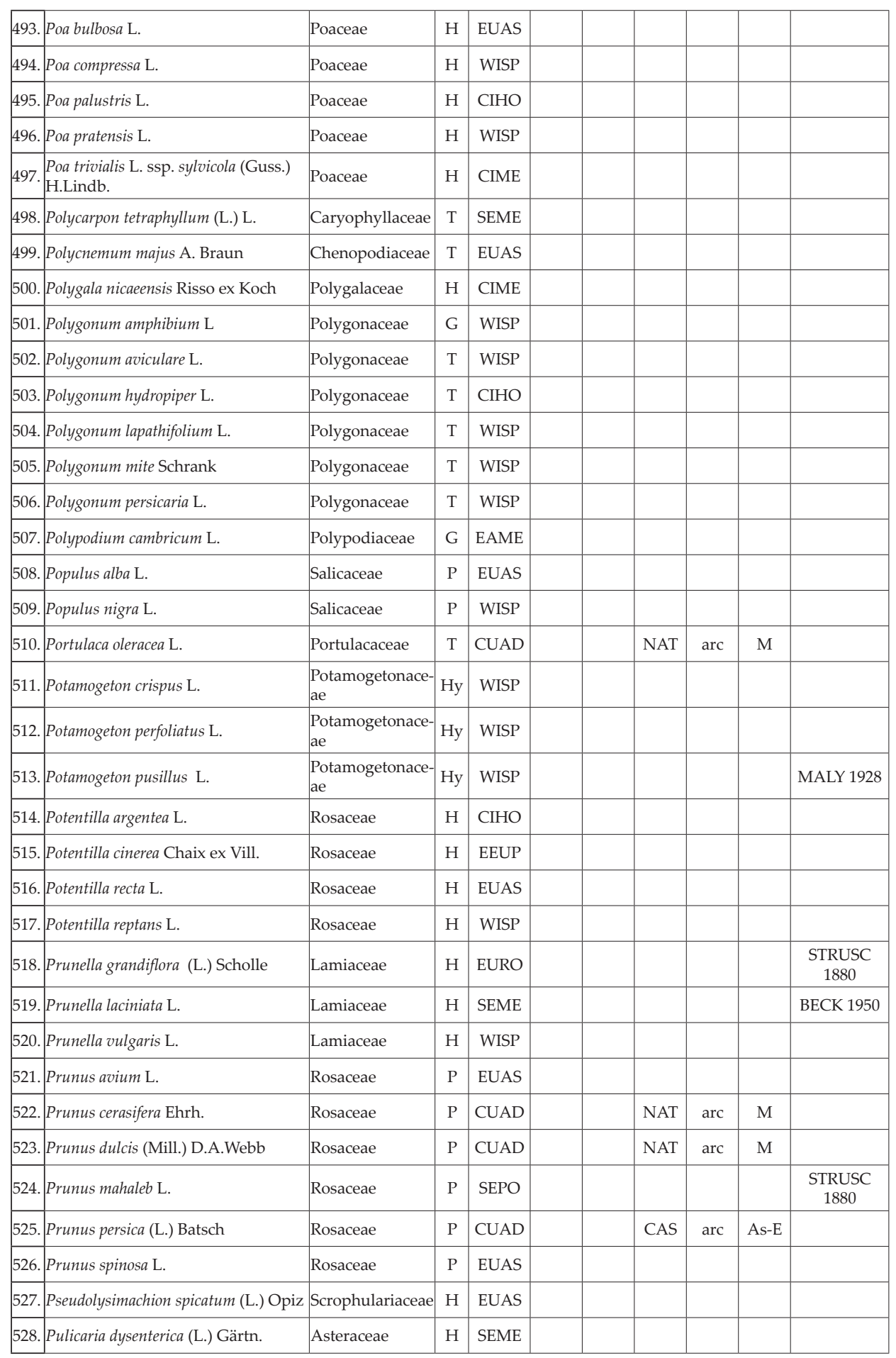




\begin{tabular}{|c|c|c|c|c|c|c|c|c|c|c|}
\hline 529.1 & Punica granatum $\mathrm{L}$ & Punicaceae & $\mathrm{P}$ & CUAD & & & NAT & $\operatorname{arc}$ & As-SW & BECK 1927 \\
\hline 530. & Pyrus amygdaliformis Vill. & Rosaceae & $\mathrm{P}$ & SEME & & & & & & \\
\hline 531.1 & Pyrus pyraster Burgsd. & Rosaceae & $\mathrm{P}$ & EUAS & & & & & & BECK 1927 \\
\hline 532. & Quercus cerris $\mathrm{L}$ & Fagaceae & $\mathrm{P}$ & EUME & & & & & & BECK 1916 \\
\hline 533. & Quercus pubescens Willd. & Fagaceae & $\mathrm{P}$ & SEPO & & & & & & $\begin{array}{c}\text { STRUSC } \\
1880\end{array}$ \\
\hline 534. & Ranunculus arvensis $\mathrm{L}$. & Ranunculaceae & $\mathrm{T}$ & CUAD & & & NAT & arc & M & \\
\hline 535. & Ranunculus ficaria L. & Ranunculaceae & G & SEME & & & & & & \\
\hline 536. & Ranunculus millefoliatus Vahl & Ranunculaceae & $\mathrm{H}$ & SEME & & & & & & \\
\hline 537.1 & Ranunculus muricatus $\mathrm{L}$ & Ranunculaceae & $\mathrm{T}$ & CIME & & & & & & \\
\hline 538. & Ranunculus neapolitanus Ten. & Ranunculaceae & $\mathrm{H}$ & SEME & & & & & & \\
\hline 539.1 & Ranunculus repens $\mathrm{L}$. & Ranunculaceae & $\mathrm{H}$ & WISP & & & & & & \\
\hline 540. & Ranunculus sardous Crantz & Ranunculaceae & $\mathrm{T}$ & WISP & & & & & & \\
\hline 541. & Ranunculus trichophyllus Chaix. & Ranunculaceae & Hy & EURO & & & & & & \\
\hline 542. & Raphanus satious L. & Brassicaceae & $\mathrm{T}$ & CUAD & & & CAS & arc & \begin{tabular}{|c|} 
Un- \\
know
\end{tabular} & \\
\hline 543. 1 & Reichardia picroides (L.) Roth. & Asteraceae & $\mathrm{H}$ & CIME & & & & & & \\
\hline 544. & Reseda lutea L. & Resedaceae & $\mathrm{H}$ & WISP & & & & & & \\
\hline 545. & Reseda phyteuma L. & Resedaceae & $\mathrm{T}$ & SEME & & & & & & \\
\hline 546. & Rhagadiolus stellatus (L.) Gaertner & Asteraceae & $\mathrm{T}$ & CIME & & & & & & \\
\hline 547 & $\begin{array}{l}\text { Rhamnus intermedius Steud. et } \\
\text { Hohst. }\end{array}$ & Rhamnaceae & $\mathrm{P}$ & ILAE & end & EN & & & & \\
\hline 548. & $\begin{array}{l}\text { Rhamnus saxatilis Jacq. subsp. } \\
\text { saxatilis }\end{array}$ & Rhamnaceae & $\mathrm{P}$ & SEMO & & & & & & \\
\hline 549. & Rhinanthus rumelicus Velen. & Scrophulariaceae & $\mathrm{T}$ & ILBE & & & & & & \\
\hline 550. & Ricinus communis $\mathrm{L}$ & Euphorbiaceae & $\mathrm{T}$ & CUAD & & & CAS & arc & \begin{tabular}{|c|} 
Un- \\
know
\end{tabular} & \\
\hline 551. 1 & Robinia pseudoacacia $\mathrm{L}$. & Fabaceae & $\mathrm{P}$ & CUAD & & & INV & neo & Am-N & \\
\hline 552. & Rorippa lippizensis (Wulfen) Rchb. & Brassicaceae & $\mathrm{H}$ & SEEU & & & & & & \\
\hline 553. 1 & Rorippa sylvestris (L.) Bess. & Brassicaceae & $\mathrm{H}$ & EUAS & & & & & & \\
\hline 554. & Rosa canina $\mathrm{L}$. & Rosaceae & $\mathrm{P}$ & WISP & & & & & & \\
\hline 555. & Rubus caesius L. & Rosaceae & $\mathrm{P}$ & EUAS & & & & & & \\
\hline 556. & $\begin{array}{l}\text { Rubus heteromorphus Ripart ex } \\
\text { Genev. }\end{array}$ & Rosaceae & $\mathrm{P}$ & ILAP & & & & & & \\
\hline 557. & Rubus ulmifolius Schott. & Rosaceae & $\mathrm{P}$ & MEAT & & & & & & BECK 1927 \\
\hline 558. & Rumex conglomeratus Murray & Polygonaceae & $\mathrm{H}$ & WISP & & & & & & \\
\hline 559. & $\begin{array}{l}\text { Rumex obtusifolius L. ssp. sylvestris } \\
\text { (Wallr.) Čelak. }\end{array}$ & Polygonaceae & $\mathrm{H}$ & EUAS & & & & & & \\
\hline 560. & Rumex pulcher $\mathrm{L}$. & Polygonaceae & $\mathrm{T}$ & SEPO & & & & & & \\
\hline 561.1 & Ruscus aculeatus L & Liliaceae & G & CIME & & VU & & & & BECK 1903 \\
\hline 562. & Ruta chalepensis L. & Rutaceae & $\mathrm{Ch}$ & CUAD & & & CAS & $\operatorname{arc}$ & M & \\
\hline 563. 1 & Ruta graveolens L. & Rutaceae & $\mathrm{Ch}$ & ILAP & & & & & & BECK 1920 \\
\hline 564. & Saccharum strictum (Host) Spreng. & Poaceae & $\mathrm{Ch}$ & EAME & & & & & & \\
\hline
\end{tabular}




\begin{tabular}{|c|c|c|c|c|c|c|c|c|c|}
\hline 565. & Salix alba $\mathrm{L}$. & Salicaceae & $\mathrm{P}$ & EUAS & & & & & \\
\hline 566. & Salvia bertolonii Vis. & Lamiaceae & $\mathrm{H}$ & ILAE & NT & & & & \\
\hline 567. & Salvia officinalis L. & Lamiaceae & $\mathrm{Ch}$ & EUME & & & & & BECK 1974 \\
\hline 568. & Salvia sclarea $\mathrm{L}$. & Lamiaceae & $\mathrm{T}$ & SEME & & & & & MALY 1928 \\
\hline 569. & Salvia verbenaca $\mathrm{L}$. & Lamiaceae & $\mathrm{H}$ & MEAT & & & & & \\
\hline 570. & Salvia verticillata $\mathrm{L}$. & Lamiaceae & $\mathrm{H}$ & SEME & & & & & \\
\hline 571. & Salvia viridis $\mathrm{L}$. & Lamiaceae & $\mathrm{T}$ & SEME & & & & & \\
\hline 572. & Sambucus ebulus L. & Caprifoliaceae & $\mathrm{H}$ & EURO & & & & & $\begin{array}{c}\text { STRUSC } \\
1880\end{array}$ \\
\hline 573. & Sambucus nigra L. & Caprifoliaceae & $\mathrm{P}$ & EURO & & & & & $\begin{array}{l}\text { STRUSC } \\
1880\end{array}$ \\
\hline 574 & Sanguisorba minor Scop. ssp. minor & Rosaceae & $\mathrm{H}$ & EUAS & & & & & \\
\hline 575. & Saponaria officinalis $\mathrm{L}$. & Caryophyllaceae & $\mathrm{H}$ & WISP & & & & & \\
\hline 576. & Satureja cuneifolia Ten. & Lamiaceae & $\mathrm{Ch}$ & ILAP & & & & & BECK 1983 \\
\hline 577. & Satureja montana L. ssp montana & Lamiaceae & $\mathrm{Ch}$ & MEPO & & & & & \\
\hline 578. & Saxifraga tridactylites $\mathrm{L}$. & Saxifragaceae & $\mathrm{T}$ & WISP & & & & & BECK 1923 \\
\hline 579. & Scabiosa triandra $\mathrm{L}$. & Dipsacaceae & $\mathrm{H}$ & SEME & & & & & \\
\hline 580. & Scandix pecten -veneris $\mathrm{L}$. & Apiaceae & $\mathrm{T}$ & WISP & & & & & \\
\hline 581. & Scilla autumnalis L. & Liliaceae & G & MEPO & & & & & FIALA 1890 \\
\hline 582. & Scilla bifolia $\mathrm{L}$. & Liliaceae & G & SEME & & & & & \\
\hline 583. & Scirpus holoschoenus L. & Cyperaceae & G & CIME & & & & & \\
\hline 584. & Scirpus lacustris L. & Cyperaceae & G & WISP & & & & & BECK 1903 \\
\hline 585. & Scolymus hispanicus L. & Asteraceae & $\mathrm{T}$ & CIME & & & & & \\
\hline 586. & Scorzonera villosa Scop. & Asteraceae & $\mathrm{H}$ & ILSE & & & & & \\
\hline 587. & $\begin{array}{l}\text { Scrophularia canina L. ssp. bicolor } \\
\text { (Sibth. et Sm.) Greuter }\end{array}$ & Scrophulariaceae & $\mathrm{H}$ & SEME & & & & & \\
\hline 588. & Secale cereale $\mathrm{L}$. & Poaceae & $\mathrm{T}$ & CUAD & & CAS & $\operatorname{arc}$ & $\begin{array}{c}\text { Un- } \\
\text { know }\end{array}$ & \\
\hline 589. & Securigera cretica (L.) Lassen & Fabaceae & $\mathrm{T}$ & EAME & & & & & \\
\hline 590. & $\begin{array}{l}\text { Securigera securidaca (L.) Deg. et } \\
\text { Dörfl. }\end{array}$ & Fabaceae & $\mathrm{T}$ & CIME & & & & & \\
\hline 591. & Sedum acre L. & Crassulaceae & $\mathrm{Ch}$ & WISP & & & & & BECK 1923 \\
\hline 592. & Sedum dasyphyllum $\mathrm{L}$. & Crassulaceae & $\mathrm{Ch}$ & SEME & & & & & BECK 1923 \\
\hline 593. & Sedum hispanicum $\mathrm{L}$. & Crassulaceae & $\mathrm{T}$ & SEPO & & & & & \\
\hline 594. & Sedum ochroleucum Chaix & Crassulaceae & $\mathrm{Ch}$ & SEME & & & & & \\
\hline 595. & Sedum sexangulare $\mathrm{L}$. & Crassulaceae & $\mathrm{Ch}$ & SEME & & & & & BECK 1923 \\
\hline 596. & $\begin{array}{l}\text { Sedum telephium L. ssp. maximum } \\
\text { (L.) Krock. }\end{array}$ & Crassulaceae & $\mathrm{H}$ & EURO & & & & & \\
\hline 597. & Selaginella denticulata (L.) Spring. & Selaginellaceae & $\mathrm{Ch}$ & MEAT & & & & & BECK 1916 \\
\hline 598. & Sempervivum tectorum $\mathrm{L}$. & Crassulaceae & $\mathrm{Ch}$ & EURO & & & & & $\begin{array}{l}\text { STRUSC } \\
1880\end{array}$ \\
\hline 599. & Senecio squalidus L. & Asteraceae & $\mathrm{H}$ & BAAP & & & & & $\begin{array}{c}\text { STRUSC } \\
1880\end{array}$ \\
\hline
\end{tabular}




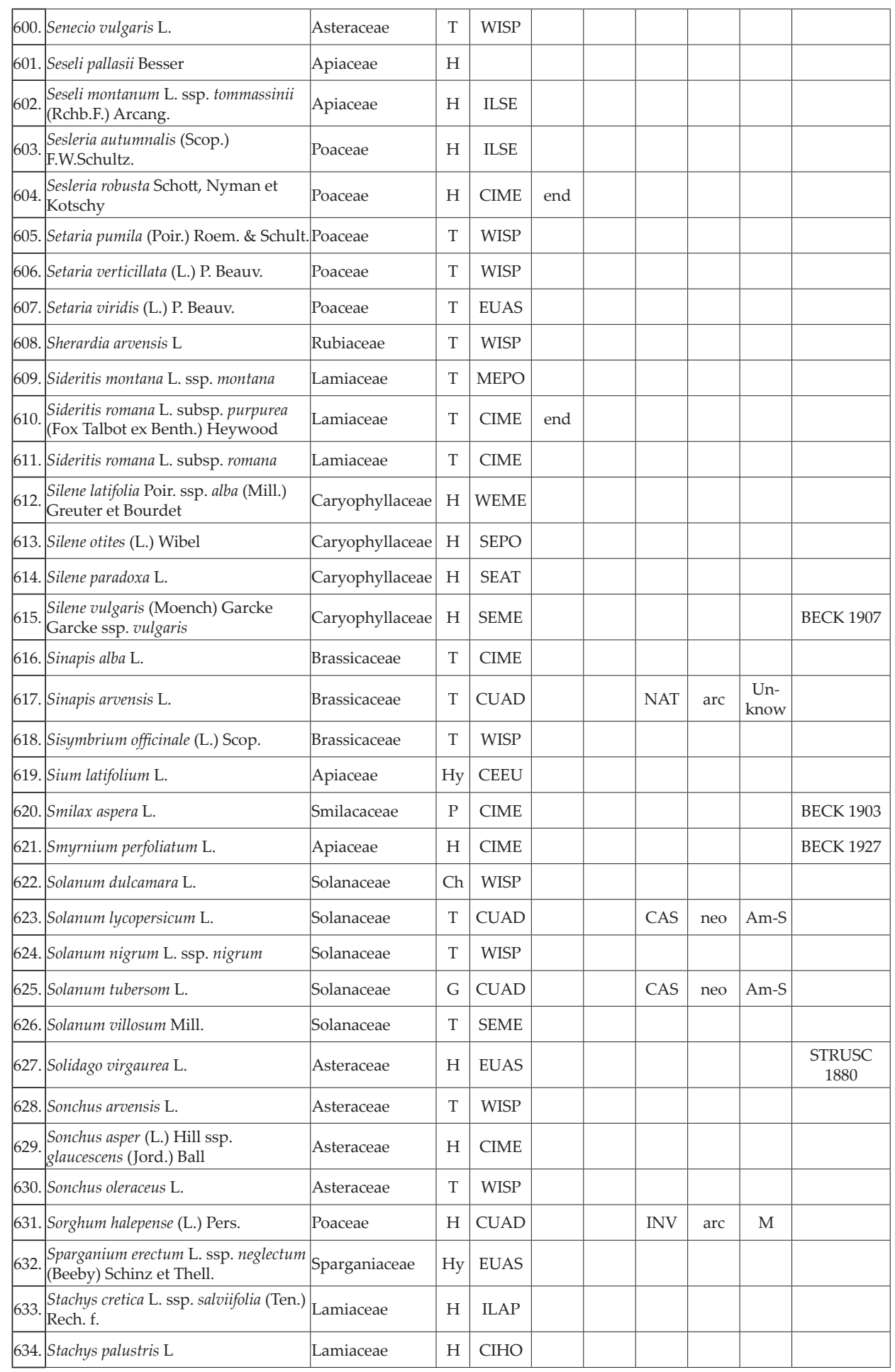




\begin{tabular}{|c|c|c|c|c|c|c|c|c|c|c|}
\hline 635. & Stachys recta $\mathrm{L}$. & Lamiaceae & $\mathrm{H}$ & SEMO & & & & & & \\
\hline 636. & Stellaria media (L.) Vill. ssp. media & Caryophyllaceae & $\mathrm{T}$ & WISP & & & & & & \\
\hline 637. & Stenbergia lutea Ker. & Amaryllidaceae & G & CIME & & $\mathrm{CR}$ & & & & \\
\hline 638. & Stipa bromoides (L.) Dörlf. & Poaceae & $\mathrm{H}$ & CIME & & & & & & \\
\hline 639. & Stipa pennata $\mathrm{L}$. & Poaceae & $\mathrm{H}$ & EUAS & & & & & & \\
\hline 640. & Symphoricarpos albus (L.) S.F.Blake & Caprifoliaceae & $\mathrm{P}$ & CUAD & & & CAS & neo & Am-N & \\
\hline 641. & Symphytum tuberosum $\mathrm{L}$. & Boraginaceae & G & SECO & & & & & & \\
\hline 642. & Tagetes minuta $\mathrm{L}$. & Asteraceae & $\mathrm{T}$ & CUAD & & & INV & neo & Am-S & ŠILIĆ, 1972 \\
\hline 643. & Tagetes patula $\mathrm{L}$. & Asteraceae & $\mathrm{T}$ & CUAD & & & CAS & neo & Am-S & \\
\hline 644. & Tamus communis $\mathrm{L}$. & Dioscoreaceae & G & SEME & & & & & & \\
\hline 645. & $\begin{array}{l}\text { Tanacetum cinerarifolium (Trev.) } \\
\text { Schultz Bip. }\end{array}$ & Asteraceae & $\mathrm{H}$ & ILAE & end & VU & & & & \\
\hline 646. & Taraxacum hoppeanum Griseb. & Asteraceae & $\mathrm{H}$ & BAAP & & & & & & \\
\hline 647. & Taraxacum officinale Webber & Asteraceae & $\mathrm{H}$ & WISP & & & & & & \\
\hline 648. & Teucrium chamaedrys L. & Lamiaceae & $\mathrm{Ch}$ & SEPO & & & & & & \\
\hline 649. & Teucrium montanum L. & Lamiaceae & $\mathrm{Ch}$ & SEME & & & & & & $\begin{array}{c}\text { STRUSC } \\
1880\end{array}$ \\
\hline 650. & Teucrium polium $\mathrm{L}$. & Lamiaceae & $\mathrm{Ch}$ & MEPO & & & & & & BECK 1950 \\
\hline 651. & Thalictrum minus L. & Ranunculaceae & $\mathrm{H}$ & EUAS & & & & & & \\
\hline 652. & Theligonum cynocrambe $\mathrm{L}$. & Theligonaceae & $\mathrm{T}$ & SEME & & & & & & \\
\hline 653. & $\begin{array}{l}\text { Thesium divaricatum Jan. ex Mert. et } \\
\text { Koch }\end{array}$ & Santalaceae & $\mathrm{H}$ & CIME & & & & & & $\begin{array}{l}\text { STRUSC } \\
1880\end{array}$ \\
\hline 654. & Thlaspi perfoliatum $\mathrm{L}$. & Brassicaceae & $\mathrm{T}$ & EUAS & & & & & & \\
\hline 655. & Thlaspi praecox Wulf. & Brassicaceae & $\mathrm{Ch}$ & ILSE & & & & & & \\
\hline 656. & Thymus longicaulis C.Presl & Lamiaceae & $\mathrm{Ch}$ & ILAP & & & & & & \\
\hline 657. & Tordylium apulum $\mathrm{L}$. & Apiaceae & $\mathrm{T}$ & CIME & & & & & & \\
\hline 658. & Tordylium maximum $\mathrm{L}$. & Apiaceae & $\mathrm{T}$ & EUME & & & & & & \\
\hline 659. & $\begin{array}{l}\text { Torilis arvensis (Huds.) LK. ssp. } \\
\text { neglecta (Schult.) Thell. }\end{array}$ & Apiaceae & $\mathrm{T}$ & SEME & & & & & & \\
\hline 660. & Tragopogon dubius Scop & Asteraceae & $\mathrm{H}$ & SEPO & & & & & & \\
\hline 661. & Tragopogon porrifolius L. & Asteraceae & $\mathrm{H}$ & CIME & & & & & & \\
\hline 662. & $\begin{array}{l}\text { Tragopogon pratensis L. ssp. orientalis } \\
\text { (L.) Celak. }\end{array}$ & Asteraceae & $\mathrm{H}$ & EUAS & & & & & & \\
\hline 663. & Tribulus terrestis L. & Zygophyllaceae & $\mathrm{T}$ & SEME & & & & & & BECK 1920 \\
\hline 664. & Trifolium alpestre $\mathrm{L}$. & Fabaceae & $\mathrm{H}$ & SEME & & & & & & BECK 1927 \\
\hline 665. & Trifolium angustifolium $\mathrm{L}$. & Fabaceae & $\mathrm{T}$ & CIME & & & & & & \\
\hline 666. & Trifolium arvense $\mathrm{L}$. & Fabaceae & $\mathrm{T}$ & EUAS & & & & & & \\
\hline 667. & Trifolium campestre Schreb. & Fabaceae & $\mathrm{T}$ & WISP & & & & & & \\
\hline 668. & Trifolium dalmaticum Vis. & Fabaceae & $\mathrm{T}$ & ILAE & end & & & & & \\
\hline 669. & Trifolium fragiferum $\mathrm{L}$. & Fabaceae & $\mathrm{H}$ & EUAS & & & & & & \\
\hline 670. & $\begin{array}{l}\text { Trifolium incarnatum L. subsp. } \\
\text { molinerii (Balb. Ex Hornem) Syme }\end{array}$ & Fabaceae & $\mathrm{T}$ & MEAT & & & & & & \\
\hline
\end{tabular}




\begin{tabular}{|c|c|c|c|c|c|c|c|c|c|}
\hline 671. & Trifolium montanum $\mathrm{L}$. & Fabaceae & $\mathrm{T}$ & EUAS & & & & & BECK 1927 \\
\hline 672. & Trifolium ochroleucum Huds. & Fabaceae & $\mathrm{H}$ & SEPO & & & & & \\
\hline 673. & Trifolium pratense L. ssp. pratense & Fabaceae & $\mathrm{H}$ & EUAS & & & & & \\
\hline 674. & Trifolium repens L. ssp. repens & Fabaceae & $\mathrm{H}$ & WISP & & & & & BECK 1927 \\
\hline 675. & Trifolium setiferum Boiss. & Fabaceae & $\mathrm{T}$ & ILAP & & & & & BECK 1927 \\
\hline 676. & Trifolium stellatum $\mathrm{L}$. & Fabaceae & $\mathrm{T}$ & CIME & & & & & \\
\hline 677. & Trigonella esculenta Willd.. & Fabaceae & $\mathrm{T}$ & EUME & & & & & \\
\hline 678. & Triticum aestivum $\mathrm{L}$. & Poaceae & $\mathrm{T}$ & CUAD & & CAS & arc & As-SW & \\
\hline 679. & Tussilago farfara $\mathrm{L}$. & Asteraceae & G & EUAS & & & & & \\
\hline 680. & Typha latifolia $\mathrm{L}$. & Typhaceae & Hy & WISP & & & & & \\
\hline 681. & Ulmus minor Miller & Ulmaceae & $\mathrm{P}$ & WISP & & & & & \\
\hline 682. & Umbilicus horizontalis (Guss.) DC. & Crassulaceae & $\mathrm{Ch}$ & CIME & & & & & MALY 1905 \\
\hline 683. & Urospermum picroides (L.) Desf. & Asteraceae & $\mathrm{T}$ & CIME & & & & & \\
\hline 684. & Urtica dioica $\mathrm{L}$. & Urticaceae & $\mathrm{H}$ & WISP & & & & & \\
\hline 685. & Urtica urens L. & Urticaceae & $\mathrm{T}$ & WISP & & & & & \\
\hline 686. & Valantia muralis $\mathrm{L}$ & Rubiaceae & $\mathrm{T}$ & CIME & & & & & \\
\hline 687. & Valeriana tuberosa $\mathrm{L}$. & Valerianaceae & $\mathrm{H}$ & SEME & & & & & \\
\hline 688. & Valerianella locusta (L.) Laterr. & Valerianaceae & $\mathrm{T}$ & CIME & & & & & \\
\hline 689. & Verbascum blattaria $\mathrm{L}$. & Scrophulariaceae & $\mathrm{H}$ & SEPO & & & & & \\
\hline 690. & Verbascum densiflorum Bertol. & Scrophulariaceae & $\mathrm{H}$ & EUME & & & & & \\
\hline 691. & Verbascum lychnitis L. & Scrophulariaceae & $\mathrm{H}$ & EURO & & & & & \\
\hline 692. & Verbascum orientale (L.) All. & Scrophulariaceae & $\mathrm{H}$ & EAME & EN & & & & \\
\hline 693. & Verbascum phlomoides L. & Scrophulariaceae & $\mathrm{H}$ & EUME & & & & & \\
\hline 694. & Verbascum pulverulentum Vill. & Scrophulariaceae & $\mathrm{H}$ & SEAT & & & & & BECK 1967 \\
\hline 695. & Verbascum sinuatum $\mathrm{L}$, & Scrophulariaceae & $\mathrm{H}$ & CIME & & & & & $\begin{array}{c}\text { STRUSC } \\
1880\end{array}$ \\
\hline 696. & Verbena officinalis L. & Verbenaceae & $\mathrm{T}$ & WISP & & & & & $\begin{array}{c}\text { MURBEC } \\
1891\end{array}$ \\
\hline 697. & Veronica anagallis - aquatica $\mathrm{L}$. & Scrophulariaceae & $\mathrm{H}$ & EURO & & & & & \\
\hline 698. & Veronica arvensis $\mathrm{L}$. & Scrophulariaceae & $\mathrm{T}$ & EUAS & & & & & \\
\hline 699. & $\begin{array}{l}\text { Veronica austriaca L. subsp. jacquinii } \\
\text { (Baumg.) Eb.Fisch. }\end{array}$ & Scrophulariaceae & $\mathrm{H}$ & EEUP & & & & & \\
\hline 700. & Veronica beccabunga $\mathrm{L}$. & Scrophulariaceae & Hy & $\mathrm{CIHO}$ & & & & & \\
\hline 701. & Veronica cymbalaria Bod. & Scrophulariaceae & $\mathrm{T}$ & SEME & & & & & \\
\hline 702. & Veronica hederifolia $\mathrm{L}$. & Scrophulariaceae & $\mathrm{T}$ & EUAS & & & & & \\
\hline 703. & Veronica persica Poir. & Scrophulariaceae & $\mathrm{T}$ & CUAD & & INV & neo & As-W & \\
\hline 704. & Veronica polita Fr. & Scrophulariaceae & $\mathrm{T}$ & EUAS & & & & & \\
\hline 705. & Veronica serpyllifolia $\mathrm{L}$. & Scrophulariaceae & $\mathrm{H}$ & WISP & & & & & BECK 1967 \\
\hline 706. & Vicia angustifolia L. ssp. angustifolia & Fabaceae & $\mathrm{T}$ & EURO & & & & & \\
\hline 707. & Vicia grandiflora Scop. & Fabaceae & $\mathrm{T}$ & EEUP & & & & & \\
\hline
\end{tabular}




\begin{tabular}{|c|c|c|c|c|c|c|c|c|}
\hline 708. & Vicia hybrida $\mathrm{L}$. & Fabaceae & $\mathrm{T}$ & CIME & & & & \\
\hline 709. & Vicia melanops Sibth. \& Sm. & Fabaceae & $\mathrm{T}$ & SEEU & & & & \\
\hline 710. & Vicia narbonensis L. & Fabaceae & $\mathrm{T}$ & CIME & & & & \\
\hline 711. & $\begin{array}{l}\text { Vicia villosa Roth ssp. varia (Host) } \\
\text { Corb }\end{array}$ & Fabaceae & $\mathrm{T}$ & EEUP & & & & \\
\hline 712. & Vinca major $\mathrm{L}$. & Apocynaceae & $\mathrm{Ch}$ & CUAD & CAS & arc & M & \\
\hline 713. & Vincetoxicum hirundinaria Medik. & Asclepiadaceae & $\mathrm{H}$ & EUAS & & & & BECK 1927 \\
\hline 714. & Viola alba Besser & Violaceae & $\mathrm{H}$ & SEME & & & & \\
\hline 715. & Viola arvensis Murr. & Violaceae & $\mathrm{T}$ & WISP & & & & \\
\hline 716. & Viola odorata $\mathrm{L}$. & Violaceae & $\mathrm{H}$ & EURO & & & & \\
\hline 717. & Viscum album L. ssp. album & Santalaceae & $\mathrm{H}$ & EUAS & & & & $\begin{array}{l}\text { STRUSC } \\
1880\end{array}$ \\
\hline 718. & Vitex agnus - castus L. & Verbenaceae & $\mathrm{P}$ & CIME & & & & $\begin{array}{l}\text { STRUSC } \\
1880\end{array}$ \\
\hline 719. & $\begin{array}{l}\text { Vitis vinifera L. ssp sylvestris (Gm.) } \\
\text { Hegi }\end{array}$ & Vitaceae & $P$ & SEME & & & & BECK 1921 \\
\hline 720. & Vulpia myuros (L.) C.C.Gmel & Poaceae & $\mathrm{T}$ & WISP & & & & \\
\hline 721. & Xantthium spinosum $\mathrm{L}$. & Asteraceae & $\mathrm{T}$ & CUAD & INV & neo & Am-S & $\begin{array}{c}\text { STRUSC } \\
1880\end{array}$ \\
\hline 722. & $\begin{array}{l}\text { Xanthium strumarium L. ssp. italicum } \\
\text { (Moretti) D.Löve }\end{array}$ & Asteraceae & $\mathrm{T}$ & CUAD & INV & neo & Am-S & \\
\hline 723. & Zea mays L. & Poaceae & $\mathrm{T}$ & CUAD & CAS & neo & Am-S & \\
\hline
\end{tabular}

